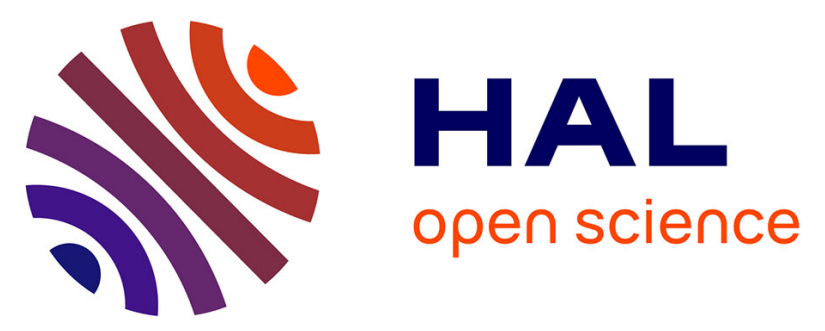

\title{
A new pancreatic adenocarcinoma-derived organoid model of acquired chemoresistance to FOLFIRINOX: first insight of the underlying mechanisms
}

Elsa Hadj Bachir, Charles Poiraud, Sonia Paget, Nicolas Stoup, Soumaya El Moghrabi, Belinda Duchêne, Nathalie Jouy, Antonino Bongiovanni, Meryem Tardivel, Louis-bastien Weiswald, et al.

\section{To cite this version:}

Elsa Hadj Bachir, Charles Poiraud, Sonia Paget, Nicolas Stoup, Soumaya El Moghrabi, et al.. A new pancreatic adenocarcinoma-derived organoid model of acquired chemoresistance to FOLFIRINOX: first insight of the underlying mechanisms. Biology of the Cell, In press, 10.1111/boc.202100003 . hal-03369738

\section{HAL Id: hal-03369738 \\ https://hal.science/hal-03369738}

Submitted on 7 Oct 2021

HAL is a multi-disciplinary open access archive for the deposit and dissemination of scientific research documents, whether they are published or not. The documents may come from teaching and research institutions in France or abroad, or from public or private research centers.
L'archive ouverte pluridisciplinaire HAL, est destinée au dépôt et à la diffusion de documents scientifiques de niveau recherche, publiés ou non, émanant des établissements d'enseignement et de recherche français ou étrangers, des laboratoires publics ou privés. 


\section{A new pancreatic adenocarcinoma-derived organoid model of acquired chemoresistance to FOLFIRINOX: first insight of the underlying mechanisms}

Elsa Hadj Bachir ${ }^{1 *}$, Charles Poiraud ${ }^{12^{*}}$, Sonia Paget ${ }^{1}$, Nicolas Stoup ${ }^{1}$, Soumaya El Moghrabi $^{1}$, Belinda Duchêne ${ }^{1}$, Nathalie Jouy $^{3}$, Antonino Bongiovanni ${ }^{3}$, Meryem Tardivel ${ }^{3}$, Louis-Bastien Weiswald ${ }^{4}$, Marie Vandepeutte ${ }^{1}$, César Beugniez ${ }^{1,2}$, Fabienne Escande ${ }^{5}$, Emmanuelle Leteurtre ${ }^{1,6}$, OrgaRES consortium ${ }^{\S}$, Laurent Poulain ${ }^{4}$, Chann Lagadec ${ }^{1}$, Pascal Pigny $^{1}$, Nicolas Jonckheere ${ }^{1}$, Florence Renaud ${ }^{1,6}$, Stephanie Truant ${ }^{1,2}$, Isabelle Van Seuningen ${ }^{1^{*}}$, Audrey Vincent ${ }^{*}$

1 Univ. Lille, CNRS, Inserm, CHU Lille, UMR9020-U1277 - CANTHER - Cancer Heterogeneity Plasticity and Resistance to Therapies, F-59000 Lille, France

${ }^{2}$ Department of Digestive Surgery and Transplantation, CHU Lille, Lille, France

${ }^{3}$ Univ. Lille, UMS 2014 - US 41 - PLBS - Plateformes Lilloises en Biologie \& Santé, Biolmaging Center Lille (BICeL), F-59000, Lille, France

${ }^{4}$ Normandie Univ, UNICAEN, Inserm U1086 ANTICIPE "Interdisciplinary Research Unit for Cancer Prevention and Treatment", 14000 Caen, France; UNICANCER, Cancer Centre F. Baclesse, 14076 Caen, France

${ }^{5}$ Department of Biochemistry and Molecular Biology, Hormonology Metabolism Nutrition Oncology, CHU Lille, F-59000, Lille, France

${ }^{6}$ Univ. Lille, Pathology Department, CHU Lille, Lille, France

$\S$ OrgaRES consortium: Christophe Mariette, Guillaume Piessen, François Corfiotti, Clarisse Eveno, François-René Pruvot, Stéphanie Truant, Mehdi El Amrani, Emmanuelle Leteurtre, Florence Renaud, Viviane Gnemmi, Laurence Wicquart, Fabienne Escande, Julie Leclerc, Sonia Paget, Soumaya El Moghrabi, Audrey Vincent, Isabelle Van Seuningen

${ }^{*}$ Both authors contributed equally to this work

${ }^{£}$ Correspondence should be addressed to:

Audrey Vincent, PhD

UMR9020 CNRS, 1277 Inserm

CANcer Heterogeneity, Plasticity and Resistance to THERapies

Mucins, Cancer and Drug Resistance

Bâtiment Cancer

Place de Verdun, 59045 Lille cedex

Tél. +33 (0)320298864 


\section{Abstract}

Background Information: Although improvements have been made in the management of pancreatic adenocarcinoma (PDAC) during the past 20 years, the prognosis of this deadly disease remains poor with an overall 5-year survival under 10\%. Treatment with FOLFIRINOX, a combined regimen of 5-fluorouracil, irinotecan (SN-38) and oxaliplatin, is nonetheless associated with an excellent initial tumor response and its use has allowed numerous patients to go through surgery while their tumor was initially considered unresectable. These discrepancies between initial tumor response and very low long-term survival are the consequences of rapidly acquired chemoresistance and represent a major therapeutic frontier. To our knowledge, a model of resistance to the combined three drugs has never been described due to the difficulty of modeling the FOLFIRINOX protocol both in vitro and in vivo. Patient-Derived tumour Organoids (PDO) are the missing link that has long been lacking in the wide range of epithelial cancer models between $2 \mathrm{D}$ adherent cultures and in vivo xenografts. In this work we sought to set up a model of PDO with resistance to FOLFIRINOX regimen that we could compare to the paired naive PDO.

Results: We first extrapolated physiological concentrations of the three drugs using previous pharmacodynamics studies and bi-compartmental elimination models of oxaliplatin and SN-38. We then treated PaTa-1818x naive PDAC organoids with six cycles of $72 \mathrm{~h}$ FOLFIRINOX treatment followed by $96 \mathrm{~h}$ interruption. Thereafter, we systematically compared treated organoids to PaTa-1818x naive organoids in terms of growth, proliferation, viability and expression of genes involved in cancer stemness and aggressiveness.

Conclusions: We reproductively obtained resistant organoids FoxR that significantly showed less sensitivity to FOLFORINOX treatment than the PaTa-1818x naive organoids from which they were derived. Our resistant model is representative of the sequential steps of chemoresistance observed in patients in terms of growth arrest (proliferation blockade), residual disease (cell quiescence/dormancy) and relapse.

Significance: To our knowledge, this is the first genuine in vitro model of resistance to the three drugs in combined therapy. This new PDO model will be a great asset for the discovery of acquired chemoresistance mechanisms, knowledge that is mandatory before offering new therapeutic strategies for pancreatic cancer. 
Abbreviations:

5-FU: 5-fluorouracil

ABCG2: ATP-binding cassette super-family $G$ member 2

CCNE1: cyclin E1

DSB: double strand break

ERK: extracellular signal-regulated kinase

FAK: focal adhesion kinase

FOLFIRINOX: Folinic acid, 5-fluorouracil, irinotecan, oxaliplatin

FoxR: Resistant organoids to FOLFIRINOX

H2AX: H2A histone family member $X$

IC50: half maximum inhibitory concentration

ITGB7: integrin subunit beta 7

LGR5: Leucine-rich repeat-containing G-protein coupled receptor 5

MRP: multidrug resistant protein

OCT4: octamer binding transcription factor 4

PARP: Poly (ADP-ribose) polymerase

PaTa: PAncreatic TAil

PDAC: Pancreatic ductal adenocarcinoma

PDO: Patient derived tumour organoid

S100A4: S100 calcium binding protein A4

SD: Standard deviation

S.E.M.: Standard Error of the Mean 


\section{Acknowledgements:}

We thank Dr Julie Leclerc, Dr Bernadette Neve and Mouloud Souidi, for thorough reading of the manuscript and for scientific discussions along the project. We thank the "Tumorothèque of the GCS-ALLIANCE CANCER of Lille" for biological resources and associated clinical data. We thank the OrgaRES core facility of the OrgaLille Platform (Univ. Lille) for 3D organoid culture. We thank Emilie Floquet (UMS 2014 - US 41 - PLBS Plateformes Lilloises en Biologie \& Santé, Biolmaging Center Lille (BICeL)) for her technical help. We thank Dr Emilie Brotin from the ImpedanCELL (real-time cellular activity) platform (CICC F. Baclesse, Caen) for real-time imaging of organoids. We thank Solène Audry from the pathology department of the Centre de Biologie Pathologie (CHU Lille).

Funding: This work was supported by academic fundings from Inserm, Université de Lille, CNRS and CHU de Lille and by grants from the SIRIC ONCOLille, Grant INCa-DGOS-Inserm 6041 (IVS), "Contrat de Plan Etat Région" CPER Cancer 2007-2013 (IVS), the Ligue Nationale contre le Cancer, comité de l'Oise and comité du Nord. This work was also supported by a grant from Contrat de Plan Etat-Région CPER Cancer 2015-2020. Sonia Paget is a recipient of Cancéropôle Nord-Ouest funding. Elsa Hadj Bachir and Nicolas Stoup are recipients of a PhD fellowship from Inserm and Région Hauts de France. 


\section{Introduction}

Given its rising incidence, pancreatic ductal adenocarcinoma (PDAC) is expected to rank as the second leading cause of cancer death in Europe by 2030 (Rahib et al., 2014). Despite tremendous efforts to improve the prognosis of this malignancy, PDAC remains the deadliest of all cancer types with a 5-year survival rate below 10\% (American Cancer Society, January 2020). Surgery is the only potentially curative treatment but $80 \%$ of patients presents with unresectable disease at diagnosis (locally advanced or metastatic) and even in cases of complete surgical resection, $90 \%$ of those patients relapse within 5 years (Vincent et al., 2011; Mizrahi et al., 2020).

Recently, a combined regimen of 5-fluorouracil, irinotecan and oxaliplatin (FOLFIRINOX) has been acknowledged in Europe as the best treatment in metastatic (Conroy et al., 2011), adjuvant (Conroy et al., 2018) and neoadjuvant settings (Murphy et al., 2018). Indeed, the response rates to FOLFIRINOX exceed greatly those of gemcitabine and allowed numerous patients with locally advanced or borderline resectable PDAC to benefit from surgery. However, despite these promising results, most patients finally relapse or progress during FOLFIRINOX treatment due to acquired chemoresistance. In fact, the development of resistance to FOLFIRINOX represents today one of the most important obstacles limiting survival in PDAC.

Numerous research teams, including ours, have developed in vitro models to decipher pancreatic cancer chemoresistance. Most of them studied gemcitabine chemoresistance (EI Amrani et al., 2019) while others examined the individual effect of the three drugs included in the FOLFIRINOX protocol (Skrypek et al., 2013, 2015) or used irinotecan instead of its active metabolite SN-38 (Romero-Calvo et al., 2019). These drugs show different impacts on DNA stability by preventing synthesis, causing damage, or inhibiting repair. To our knowledge, no in vitro model of sustained PDAC resistance to the combined drugs of the FOLFIRINOX protocol has been published probably due to the difficulty of modeling the FOLFIRINOX protocol both in vitro and in vivo. Therefore, mechanisms underlying this phenomenon are largely unknown.

Patient-derived tumour organoids (PDO) are a novel pertinent model to study cancer resistance as they maintain key features of the tumour they originate from (Baker et al., 2016; Nagle et al., 2018; Pasch et al., 2019). Hence, these past few years, the usefulness of PDAC organoids was demonstrated to identify molecular pathways that could represent therapeutic opportunities (Boj et al., 2015). These in vitro models have shown a strong reliability to decipher cellular mechanisms involved in response to therapy and represent the 
missing link between 2D cell line cultures and in vivo xenografts (Bleijs et al., 2019). Tumour organoids can be cultured from surgically resected tumors as well as from endoscopic biopsies using fine-needle aspirates (Boj et al., 2015) and even more recently from circulating tumor cells (Praharaj et al., 2018). In the present study, we used PDAC organoids to set up the first in vitro model of acquired resistance to FOLFIRINOX. This new model brings the first insight into the mechanisms underlying acquired chemoresistance to FOLFIRINOX in PDAC. 


\section{Results and Discussion}

\section{Histological characterization of patient-derived pancreatic adenocarcinoma organoids}

PaTa-1818x [KRAS p.Gly12Asp (G12D, c.35G>A); TP53 p.Arg282Trp (c.844C>T)] were derived from an 85-year-old male with a moderately differentiated adenocarcinoma of the pancreatic tail (PaTa) histologically diagnosed as pT2N1M0 and naive from chemotherapy.

Histological examination of the primary tumor revealed a moderately differentiated adenocarcinoma with tubular architecture composed of glands of various sizes as classically observed in PDAC either isolated or organized in cribriform structures. Three-dimensional clusters as well as isolated tumor cells with hyperchromatic nuclei, irregular nucleoli, and high nuclear to cytoplasmic ratio were also observed.

Tumor cells were surrounded by a marked desmoplastic reaction and perineurial invasion.

Interestingly, this tumor presented heterogeneity (Figure 1A-C and $1 \mathrm{G}-\mathrm{I}$ ) with cancer cells of various sizes and types, including mucin-producing cells highlighted by alcian blue staining (Figure 1G-I), signet ring cells (Figure 1C and 1I, black arrows) as well as more typical cubic cells.

PaTa-1818x organoids derived from this tumor reflected the same tumor heterogeneity even after $>10$ passages in culture (Figure $1 \mathrm{D}-\mathrm{F}$ and $\mathrm{J}-\mathrm{L}$ ) with both cylindric and mucinproducing cells (Figure 1J-L) or signet-ring cells (black arrows) found in all tumor organoids. Tubular architecture was also found similar in organoids compared to the tumor of origin. Thus, PaTa-1818x organoids truly recapitulate the tumor cell architecture and heterogeneity of the pancreatic adenocarcinoma they originate from.

\section{In vitro modelling of the FOLFIRINOX regimen given to patients with pancreatic ductal adenocarcinoma}

FOLFIRINOX regimen is a combination of three cytotoxic drugs (i.e. 5-fluorouracil, irinotecan and oxaliplatin). Irinotecan is a prodrug that must be hydrolyzed by carboxyl esterase in the liver and the intestine to generate the active metabolite SN-38 (Ahmed et al., 1999; De Man et al., 2018). Thus, irinotecan cannot be used as such in vitro and must be replaced by SN-38 to reliably model in vivo protocols.

In order to accurately model the FOLFIRINOX regimen in vitro, it was first mandatory to estimate the concentration of each of the three drugs in the tumour microenvironment during a chemotherapeutic protocol. Indeed, the direct use of concentrations received by patients would be irrelevant given their intravenous administration. Therefore, we used a pharmacokinetic approach to make tissue concentration estimates, relying on their bi- 
compartmental distribution following intravenous administration. Previous studies had established that dynamics of oxaliplatin and SN-38 plasmatic concentrations respond to a biexponential equation (Mathijssen et al., 2001; Valenzuela et al., 2011). In other words, following the administration of these two drugs, plasmatic concentrations are characterized by curves with first a highly negative slope, followed by a dramatic decrease of the value of this slope (Figure 1M). The first phase is due to distribution of the drug into the tissues along with elimination. Secondly, the flattening of the plasmatic concentration curve represents pure elimination. In between, plasma and tissue concentrations reach equilibrium before slowly decreasing together. Hence, the concentration at which the value of the curve slope dramatically changes can be used as an estimate of the maximal tissue concentration of the drug. We used this principle to graphically estimate the average concentrations of oxaliplatin and $\mathrm{SN}-38$ received by the tumour during the FOLFIRINOX protocol from previously published measurements of plasmatic concentrations recently gathered in Laure Deyme et al., 2019.

Finally, in the most recent mFOLFIRINOX (modified) protocol (Conroy et al., 2018), unlike oxaliplatin and irinotecan that are administered through rapid perfusion, 5-FU is given over 46 hours through an ambulatory infusor. This ensures a stable 5-FU plasma concentration through the treatment. We therefore extrapolated 5-FU plasma concentration as the mean tissue concentration of this drug during the treatment.

Hence, concentrations of 5-FU, oxaliplatin and $\mathrm{SN}-38$ used to generate resistant organoids and subsequently to re-challenge organoids to FOLFIRINOX were $4 \mu \mathrm{M}, 0.5 \mu \mathrm{M}$ and $12.5 \mathrm{nM}$, respectively.

\section{Acquisition of pancreatic adenocarcinoma-derived organoids resistant to the FOLFIRINOX regimen}

To mimic the FOLFIRINOX protocol given to PDAC patients, PaTa-1818x were treated by six cycles of $72 \mathrm{~h}$ of $5-\mathrm{FU}$, oxaliplatin and $\mathrm{SN}-38$ at the concentrations indicated above, followed by 4 days without treatment (Figure $1 \mathrm{~N}$ ). Along the process, partial loss of organoid integrity was observed, accompanied with increased apparition of invading cells that protruded out of the organoids and even out the Matrigel domes in which they were cultured, forming larger clusters of two-dimensional cell layers compared to untreated organoids (Figure 2A, black arrows). Interestingly, FOLFIRINOX treatment of organoids in early phase of their growth (10 days after seeding) led to a different phenotypic response with rapid and abundant cell mortality resulting in isolated persistent cell clusters (Supplemental figure 1B, black arrows) at the expense of almost all organoid structures. 
Organoids that survived the six cycles of treatment were called FoxR. The process of acquisition of resistance was repeated at least three times either in organoids in their early or late phase of culture growth and led to organoids named FoxR1 to foxR7.

Analysis of genetic alteration using an optimized targeted NGS panel did not reveal any additional mutation after the six cycles of treatment. Moreover, no significant differences were observed in allele frequencies between naive PaTa-1818x (KRAS p.Gly12Asp 50\%; TP53 p.Arg282Trp 95\%) and resistant FoxR1 (p.Gly12Asp 51\%; TP53 p.Arg282Trp 98\%) organoids, indicating that repeated exposure to FOLFIRINOX did not lead to clonal expansion of underrepresented clones in our model.

In order to characterize the evolution of apoptosis and proliferation during the process of acquisition of resistance, we systematically performed cell cytometry to analyze the cell cycle status in the population before and after each FOLFIRINOX treatment (Figure 2B-C). Immediately after the first treatment with FOLFIRINOX, we observed an accumulation of the cells in the S phase (mean percentage of $36 \%$ vs. $4 \%, p<0.01$ ) while the proportion of cells in G0/G1 decreased $(83 \%$ vs. $45 \%, p<0.05)$ in treated compared to control untreated organoids. The proportion of cells in the G0/G1 phase further decreased along the second and third treatment to reach a mean of $26 \%$ but increased again after the fourth treatment (mean: $46 \%$ ). The proportion of cells in the G2/M phase was increased after the second treatment (mean: $21 \%$ ) and remained elevated compared to the control (mean: $9 \%, p<0.05$ ) along the whole process. Accumulation of cells in the $S$ and $\mathrm{G} 2 / \mathrm{M}$ phases of the cell cycle is a commonly observed response to chemotherapy and especially with 5-FU. It has been suggested that this slowdown in cell cycle prevents incorporation of 5-FU metabolites into DNA, providing cancer cells with sufficient time to correct the mis-incorporated nucleotides and therefore leading to resistance (Guo et al., 2008). In our model, patient-derived organoids rapidly adopt this mechanism but seem to quickly evolve through the cell cycle again after the $3^{\text {rd }}$ treatment, suggesting that resistance is then already acquired. And indeed, the SubG1 population, reflecting cell apoptosis, systematically reached its maximum value (12 to $20 \%$ ) between the 2 nd and 3 rd treatment with FOLFIRINOX then slowly decreased until the end of the sixth cycle of treatment, reaching values between 3 and $8 \%$.

It is important to note that SubG1 populations as well as cells accumulating in the $S$ and G2/M phase represent a maximum of $83 \%$ of the cell population treated with FOLFIRINOX, meaning that a non-negligible proportion of the cancer cells cultured as organoids do not respond to the treatment and may reflect innate resistance, while cells going through cell cycle blockade may reflect acquired resistance.

Along the process of acquired chemoresistance, we also observed the emergence of a polyploid cell population that reached a maximum mean percentage right after the $4^{\text {th }}$ cycle and before the 5 th $\left(20\right.$ to $24 \%$ ) and then decreased to an average of $13 \%$ after the $6^{\text {th }}$ 
treatment. In cancer cells, aborted mitosis is associated with reversible polyploidy (Erenpreisa \& Cragg, 2013). This mechanism is part of cancer cell escape from chemotherapy-induced genotoxic damage, particularly in a mutated TP53 environment such as PaTa-1818x organoids. Reversible polyploidy is also associated with reversible senescence and stemness and therefore, these mechanisms of resistance should be further investigated in this model.

Interestingly, when the process was started in the early phase of organoid growth (only 10 days after cell seeding), response to FOLFIRINOX treatment was widely different in terms of distribution of cell populations in the cell cycle (Supplemental figure 1C). Indeed, the subG1 apoptotic cell population was increased earlier (mean of $14 \%$ before the beginning of the $2^{\text {nd }}$ treatment) and remained high until the end of the $4^{\text {th }}$ FOLFIRINOX treatment, even reaching a peak of cell mortality of $44 \%$ before the $3^{\text {rd }}$ treatment. The blockade in the $S$ and G2/M phases was also maintained longer with $27 \%$ cells still in the G2/M phase at the end of the $6^{\text {th }}$ cycle while the proportion of cells in the G0/G1 phase was still very low at the end of the process (mean: $22 \%$ ). This blockade was accompanied with an important percentage of cells exiting the cell cycle (G0, $54.55 \%$ vs. $17.17 \%, p<0.05$, in untreated control organoids) and remaining quiescent for a long period of time (several weeks) even after the end of the $6^{\text {th }}$ treatment with FOLFIRINOX before they started growing again (data not shown). Dormant cells contribute to residual disease in many types of cancers. In pancreatic adenocarcinoma, residual disease after treatment is a major issue and usually induces cancer relapse very early after initial response to treatment. Therefore, our model would be of great interest to better understand the mechanisms of tumor dormancy and rapid relapse in PDAC. On the contrary, the percentage of cells in G0 in mature organoids after the 6 cycles of treatment was lower (29.29\%) and not significantly different from control untreated organoids. Moreover, regrowth was observed immediately after the $6^{\text {th }}$ cycle. In this format, our model would thus rather mimic partial response to treatment as observed in $30 \%$ of patients with metastatic PDAC treated with FOLFIRINOX (Conroy et al., 2011).

Finally, the polyploid cell phenotype was also not fully reversed after the $6^{\text {th }}$ cycle of treatment in early organoid cultures and rather stagnated after the $3^{\text {rd }}$ cycle with a mean percentage always higher than $22 \%$.

Taken together, these data suggest that response to FOLFIRINOX in early growing organoids is more acute than in fully grown organoids, with more cells prone to block or exit the cell cycle and enter into apoptosis in early cultures while more cells are able to quickly resolve these phases in late cultures. Many parameters that are different in early vs. late 3D culture may explain these results: stemness vs. differentiation balance, more active proliferation rate, cell organization as complex 3D structures, hypoxia gradients. Therefore, 
depending on the way this model is used for future studies, it may be suited for exploration of diverse aspects of chemoresistance.

\section{Expression profiles of key genes are modified through the acquisition of resistance to FOLFIRINOX}

In order to identify key markers of resistance acquisition to FOLFIRINOX, gene expression analysis was performed across treatment cycles and systematically compared between FOLFIRINOX-treated and naive PaTa-1818x (Figure 3A and Supplemental Figure 2).

When we looked at genes implicated in cell proliferation, we observed an increase of $c$ myc and CCNE1 (encoding Cyclin E1) gene expression right after the first $72 \mathrm{~h}$ of FOLFIRINOX treatment (by 1.8- and 2.1-fold, respectively) although this was not statistically significant. C-myc transient overexpression was observed only during the first cycle of treatment. On the contrary, CCNE1 expression was significantly higher in treated compared to untreated PaTa-1818x after the $3^{\text {rd }}$ cycle (d17, 3.36-fold) and reached 3.96-fold and 2.65fold right after the $4^{\text {th }}$ and $5^{\text {th }}$ cycle, respectively. Interestingly, these phases of high expression of CCNE1 were alternated with phases of lower expression before the $4^{\text {th }}$ and after the $5^{\text {th }}$ cycle of treatment (Supplemental Figure 2A). Since it is known that cyclin E1 transcript levels peak in G1-S phase and are downregulated as cells enters $G 2 / M$ in particular in pluripotent stem cells (Soledad et al., 2018), the fluctuation of its expression through acquisition of resistance may reflect the progression of FOLFIRINOX-treated organoids through cell cycle, despite the chemotherapeutic treatment. Indeed, our cell cycle analysis revealed that G2/M blockade induced by FOLFIRINOX treatment was almost entirely resolved after the $4^{\text {th }}$ cycle of treatment (Figure $2 \mathrm{~B}-\mathrm{C}$ ). This indicates that chemoresistance mechanisms and sustained proliferation under treatment may emerge as soon as the $3^{\text {rd }}$ cycle of treatment.

High expression of multidrug resistance protein MDR1 (P-glycoprotein/ABCB1), MDRrelated proteins MRP1/2 (ABCC1/2), or ATP-binding cassette transporter ABCG2 (BCRP) expression (Rosner et al., 2008) has been associated with irinotecan/SN-38 chemoresistance (Skrypek et al., 2015). These transporters play an essential physiological role by exporting toxic xenobiotics, thereby reducing the efficiency of clinical drugs (Sharom, et al., 2008). Additionally, some of these transporters are potent markers of cancer stemness (Begicevic and Falasca, 2017) and are associated with more aggressive forms of epithelial cancers (Muriithi et al., 2020). Our results show that treated organoids express significantly higher levels of $A B C G 2$ compared to untreated PaTa-1818x organoids after the $2^{\text {nd }}$ and the $3^{\text {rd }}$ cycle of FOLFIRINOX treatment (2.69- and 6.7-fold, respectively). This differential expression was maintained through the $4^{\text {th }}, 5^{\text {th }}$ and $6^{\text {th }}$ cycle where it reached 4.17-, 6.74- 
and 4.82-fold, respectively. In addition, we observed a significant increase of MRP4 expression in treated compared to untreated organoids after the $4^{\text {th }}$ cycle (3.69-fold). However, this increase was not maintained through the end of the experiment. A consistent but not significant increase of MRP1 and MRP5 expression was observed before the $4^{\text {th }}$ cycle where it reached 3.05- and 2.67-fold. Hence, only accumulation of ABCG2 transcripts was sustained and seems to be associated with acquisition of resistance to FOLFIRINOX. This transporter has been shown to export cellular SN-38 in other models of acquired resistance and especially in breast cancer (Chen et al., 2019). However, although it is known to be expressed in normal pancreatic stem cells (Sasaki et al., 2018), the role of ABCG2 has never been directly linked to PDAC chemoresistance. Further studies will therefore be necessary to validate its active role in PDO acquired resistance to FOLFIRINOX. On the other hand, the pivotal role of ABCG2 in tissue protection against xenobiotics renders this transporter difficult to target, even though potent inhibitors have been identified these past few years (Toyoda et al., 2019). An interesting strategy would be to identify and reverse the mechanisms involved in its increased expression upon acquisition of resistance. Indeed, $A B C G 2$ is known to be epigenetically regulated in cancer (Turner et al., 2006; Moon et al., 2015; Spitzwieser et al., 2016) and epigenetic strategies could then be an interesting therapeutic strategy to circumvent $A B C G 2$ overexpression in resistant pancreatic cancer cells.

As stemness is associated with chemoresistance (Ercan et al., 2017), we assessed the expression of LGR5, OCT4 and NANOG pluripotency genes through the six cycles of treatment with FOLFIRINOX. No significant increase of expression of OCT4 and NANOG in treated vs untreated PaTa-1818x was detected after six cycles of FOLFIRINOX treatment (Figure $3 A$ ). Since we only used bulk organoid cultures to study gene expression, the effect of FOLFIRINOX on the stem cell compartment may be diluted in our RT-qPCR experiments. However, by using another marker of stemness in the digestive tract LGR5 (Leushacke \& Barker, 2012), we found a significant increase (8.56-fold) after the $3^{\text {rd }}$ cycle of treatment that was initiated, although not significantly, on d14 (four days after the end of the $2^{\text {nd }}$ cycle, supplemental figure 2). The role of LGR5 as a marker of cancer stem cells in PDAC has been controversial (Andrikou et al., 2015 ; Amsterdam et al., 2013). However, in our study, its synchronized increased expression with CCNE1 at the end of the $3^{\text {rd }}$ cycle, when cell accumulation in $\mathrm{S}$ and G2/M phase is resolved, suggest a role of this marker, and possibly of the Wnt pathway, in the acquisition of resistance to FOLFIRINOX.

Finally, we analyzed the expression of genes implicated in pancreatic cancer cell aggressiveness. Notably, the integrin encoding gene ITGB7 is associated with pancreatic cancer cell invasion properties (Sun et al., 2020). Interestingly, we found that ITGB7 was characterized by cyclic expression upon repeated exposure to FOLFIRINOX (Supplemental 
Figure 2), with the highest peaks reaching 3.41- and 2.68-fold expression in treated compared to untreated PaTa-1818x before the $4^{\text {th }}$ and $6^{\text {th }}$ cycle of treatment. Interestingly, these time points corresponded to the moment when maximum mortality was induced by the previous treatment, respectively the $3^{\text {rd }}$ and the $5^{\text {th }}$ cycles. Increased expression of the ITGB7 protein in treated compared to naive organoids was confirmed using Western blotting after the $4^{\text {th }}$ cycle of treatment (2.29-fold). High expression of ITGB7 after the $3^{\text {rd }}$ cycle may therefore reflect one of the key mechanisms of acquired resistance to FOLFIRINOX. Since Reactive Oxygen Species (ROS) play a key role in 5-FU, Oxaliplatin and SN-38-mediated anti-tumor effects and have been shown to activate ITGB7 transcription in pancreatic cancer cells, we investigated mitochondrial superoxide production during the process of acquired resistance to FOLFIRINOX. Our data show a peak of ROS production after the $2^{\text {nd }}$ cycle of treatment that is not observed afterwards (Figure $3 \mathrm{C}$ ). In organoids treated in their early phase of growth, this peak exactly precedes the peak of ITGB7 overexpression (supplemental figure 2B). In fully grown organoids, the delay between culmination of FOLFIRINOX-induced ROS production and increased expression of ITGB7 may indicate additional mechanisms responsible for its overexpression.

S100A4 overexpression through DNA hypomethylation has been associated with invasive and high-grade pancreatic carcinoma (Rosty et al., 2002; Lee et al., 2014; Nastase et al., 2016). Expression of this calcium-binding protein increases pancreatic cancer cell resistance to gemcitabine (Mahon et al., 2007). In our study, S100A4 expression increased progressively but not significantly between the $2^{\text {nd }}$ and the $5^{\text {th }}$ cycle, reaching 2.43 -fold and subsequently decreased to reach initial expression levels before the $6^{\text {th }}$ cycle (Supplemental Figure 2A).

Our data suggest that ITGB7 may be implicated in the early phase of acquisition of resistance to FOLFIRINOX but not in its maintenance.

In conclusion, $A B C G 2$ was the only gene in our study that responded to the criteria of an ideal chemoresistance marker with increased expression that is maintained across the successive cycles of FOLFIRINOX treatment.

Strikingly, the same gene expression changes observed along the acquisition of resistance were found when we started the FOLFIRINOX treatment of organoids in their early phase of growth (supplemental figure 2B), although these changes happened sooner (at the end of the first cycle of treatment) to the point that they had recovered initial expression rates right at the time where gene expression culminates in organoids treated in their later phase of growth (after the end of the $3^{\text {rd }}$ cycle of treatment). The lack of statistical significance probably relies on higher cell heterogeneity observed in early compared to late organoid culture phases. Also, overexpression of MRP4 seemed to be maintained along the process while it was not in late organoids (supplemental figure $2 \mathrm{~A}$ ). 


\section{FoxR organoids maintain acquired resistance to FOLFIRINOX}

In order to assess the stability of FoxR1 organoid resistance to FOLFIRINOX, we rechallenged them after several passages with a unique cycle of $72 \mathrm{~h}$-FOLFIRINOX treatment and compared their response to that of treated PaTa-1818x up to 10 days after the treatment ended (Figure 4).

Delayed cell mortality with loss of organoid integrity was detected using time-lapse imaging 24h after FOLFINOX treatment ended in FoxR1 organoid populations, whereas cell mortality was already observed in PaTa-1818x organoid populations during the treatment (Figure 4A, and Supplemental Movies 1 and 2). On d6 (48h post-treatment) organoid viability was significantly higher in FoxR1 treated compared to PaTa-1818x treated organoid populations (68.7\% vs $29.9 \%$ respectively, Figure $4 \mathrm{~B})$. This significant difference in organoid viability between FoxR1 and PaTa-1818x was maintained throughout the experiment (2-way ANOVA, $p<0.001)$ up to d 13 (43.97\% vs $18.82 \%$ respectively).

The cytostatic effect of FOLFIRINOX treatment was also higher in PaTa-1818x than in FoxR1 organoid populations. Indeed, PaTa-1818x organoid growth was quickly arrested after the beginning of FOLFIRINOX treatment. Stagnation of organoid average size occurred as soon as the treatment began and was followed by a decrease from d5 to d12 (from $7764 \mu \mathrm{m}^{2}$ to $3873 \mu \mathrm{m}^{2}$, Figure $4 \mathrm{C}$ ). Conversely, the average size of live organoids from FoxR1 populations started to decrease at $\mathrm{d} 2$ (after $24 \mathrm{~h}$ of treatment) until $\mathrm{d} 5$ and was stabilized until the end of the experiment (d13) (from $10634 \mu \mathrm{m}^{2}$ to $8736 \mu \mathrm{m}^{2}$, Figure $4 \mathrm{C}$ ). Hence, although the average size of organoids was not significantly different at the end of the treatment $(\mathrm{d} 4)$ between FoxR1 and PaTa-1818x, FoxR1 organoids were significantly larger than PaTa$1818 x$ five days after the treatment ended $(d 9, p<0.05)$ and thereafter $(p<0.01)$. Interestingly, growth ratios were very similar between FoxR1 and PaTa-1818x during FOLFIRINOX treatment (Supplemental Figure 3). However, while FoxR1 organoid growth rate started to increase slightly $72 \mathrm{~h}$ after the treatment, $\mathrm{PaTa}-1818 \mathrm{x}$ growth rate pursued its decrease.

Size repartition of live organoids in treated PaTa-1818x was also affected as observed by the shape of violin plots in Figure 4D (thin-elongated to broad-short). In tumor organoid culture, size heterogeneity reflects the wide variety of proliferation/differentiation potential between cancer cell populations through organoid morphogenesis (Hof et al., 2021 ; Shin et al., 2020). However, at the end of the experiment, standard deviation (sd) of PaTa-1818x organoid areas was only $2696 \mu^{2}$. This was particularly the case for cystic organoids which present higher proliferation rates and simpler structures. Interestingly, mature organoid size was a lot less altered by FOLFIRINOX treatment (data not shown). This may be due to inferior proportion of proliferating cells in this type of organoids and more dense 3D structures that protect inner cells from chemotherapy. On the contrary, organoid size 
heterogeneity of FoxR1 populations was overall maintained throughout the experiment and was not altered by the treatment (sd: $11610 \mu \mathrm{m}^{2}$, Figure 4D).

In order to investigate whether FoxR organoids were less sensitive to FOLFIRINOXinduced apoptosis, we analyzed Caspase 3 cleavage by western blotting (Figure 5B). We were not able to observe Caspase 3 cleavage until d5 (24h after the treatment ended) in FoxR organoids while it appeared in $\mathrm{PaTa}-1818 \mathrm{x}$ treated organoids as soon as $\mathrm{d} 4$ (right at the end of FOLFIRINOX treatment).

Next, we examined PARP-1 cleavage as an early indicator of chemotherapy-induced apoptosis generated by caspases 3 and 7 (Kaufmann et al., 1993; Amours et al., 2001), in treated FoxR and PaTa-1818x organoids. We observed PARP-1 cleavage in PaTa-1818x organoids as soon as $24 \mathrm{~h}$ after the beginning of FOLFIRINOX treatment (d2, Figure 5B). This cleavage was intensified and maintained throughout the experiment until d10 (6 days after the end of FOLFIRINOX treatment). Conversely, PARP cleavage was only very weakly initiated by FOLFIRINOX treatment on $\mathrm{d} 10$ in FoxR organoids.

Our results indicate that FOLFIRINOX treatment induces a delayed and weaker apoptosis signal in FoxR compared to PaTa-1818x organoids. These data are consistent with reduced mortality rate observed in FoxR1 compared to PaTa-1818x following FOLFIRINOX treatment (Figure 4B).

Phosphorylation of $\mathrm{H} 2 \mathrm{AX}(\gamma \mathrm{H} 2 \mathrm{AX})$ is generally used to detect the genotoxic effect of chemotherapies that induce DNA double strand breaks (DSBs) such as oxaliplatin (Podhorecka et al., 2010). While similar rate of $\gamma \mathrm{H} 2 \mathrm{AX}$ was observed $24 \mathrm{~h}$ after the beginning of FOLFIRINOX treatment in PaTa-1818x and FoxR organoids (2.8-fold vs 2.2-fold compared to respective untreated organoids, Figure 5B), a gradual amplification of the signal was observed only in PaTa-1818x through the time course of the experiment (5.65-fold to 8.7-fold on $\mathrm{d} 4$ and $\mathrm{d} 6$, respectively) and not in FoxR. On d10, $\gamma \mathrm{H} 2 \mathrm{AX}$ level decreased both in PaTa-1818x and in FoxR compared to d6 (0.73-fold vs 0.25 -fold, respectively), indicating that the maximum effects of the treatment had been reached. Phosphorylation of H2AX is required for activation of checkpoint proteins which arrest the cell cycle progression until DSBs repair or induction of apoptosis. Interestingly, we observed that FoxR1 organoid growth was arrested until d10 but was re-induced right after this time point (Supplementary figure 3).

Altogether, these data suggest that FoxR are able to signal chemotherapy-induced DNA damage through a weaker phosphorylation of $\mathrm{H} 2 \mathrm{AX}$ compared to PaTa-1818x. This might be the consequence of $A B C G 2$ overexpression in FoxR that allows the cells to pump the drug out. Additionally, unlike naive organoids, only a minority of FoxR organoids respond to $\gamma \mathrm{H} 2 \mathrm{AX}$ signal through apoptosis. Instead, they rapidly re-enter the cell cycle after 
FOLFIRINOX treatment. Further analysis will be necessary to understand if FOLFIRINOX induces less DNA damage, if DNA damage is more rapidly repaired or if apoptosis signaling is rather impaired in FoxR organoids compared to PaTa-1818x.

Finally, determination of the half-maximal inhibitory concentration (IC50) revealed that FoxR1 were indeed more resistant to higher concentrations of FOLFIRINOX than PaTa1818x organoids. Indeed, $50 \%$ cell mortality was obtained at concentrations of $0.782 \mu \mathrm{M}$ of $5-\mathrm{FU}, 0.098 \mu \mathrm{M}$ of oxaliplatin and $2.44 \mathrm{nM}$ of SN-38 for FoxR1 vs respectively $0.294 \mu \mathrm{M}$, $0.037 \mu \mathrm{M}$ and $0.92 \mathrm{nM}$ for PaTa-1818x organoids (Figure $5 \mathrm{C}$ ).

Altogether, these data validate our in vitro model consisting of repeated and alternated exposure to FOLFIRINOX treatment in order to induce chemoresistance in PDAC-derived organoids.

FoxR1 are characterized by different organoid growth, organization and stemness properties compared to PaTa-1818x organoids

FoxR1 organoid growth compared to naive PaTa-1818x was evaluated using time-lapse imaging.

Naive PaTa-1818x are characterized by early cyst formation (white arrows) which border starts thickening (intermediate state, grey arrows) and then folding and budding after several days in culture (black arrows, Figure 6A and supplemental movie 3). Budding cyst-like PaTa1818x organoids are comparable to classical organoids obtained from pancreatic small duct fragments (Huch et al., 2013). This is consistent with its origin: a moderately differentiated pancreatic adenocarcinoma. The doubling time of organoid size was approximately 4.5 days. Of note, time-lapse imaging allowed us to observe pulsatile organoid growth (Figure 6B and supplemental movies 3 and 4 ).

Eleven days after seeding of individual cells, FoxR1 organoids were larger in size than PaTa-1818x (Figure $6 \mathrm{~A}$ and 6B, supplemental movie 4). The doubling time of FoxR1 organoids was shorter than that of PaTa-1818x (approximately 3.8 days). The difference in organoid size was maintained until d18.8 (average area of $31540 \mu \mathrm{m}^{2}$ vs $21268 \mu^{2}$ respectively) where it reached its highest significance $(p<0.01$, Figure $6 \mathrm{~B})$. Organoid size is highly dependent on cell proliferation at the initiation of 3D culture. In the organoid hierarchy, cells with progenitor properties are the most highly cycling cells immediately followed by stem-like cells (Beumer and Clevers, 2016). FoxR1 organoids may thus be enriched in highly proliferating undifferentiated cells.

After d18.5, FoxR1 organoid average size slightly decreased to reach that of PaTa-1818x naive organoids at $\mathrm{d} 21\left(20533 \mu \mathrm{m}^{2}\right.$ vs $20070 \mu \mathrm{m}^{2}$, respectively). This time point corresponds to the maturation of the whole population into budding organoids and dramatic decrease in round-shaped cystic structures. Interestingly, mature organoids from FoxR1 populations 
presented more complex structures with higher folding and increased budding compared to PaTa-1818x (Figure 1N and 6A). In hierarchical organoid structuring, stem cells have been shown to be enriched at the tip of organoid buds (Loomans et al., 2018). FoxR1 organoids may then again present a higher proportion of functional stem-like cells that cluster together, leading to higher 3D structuration than in naive organoids.

We thus compared the percentage of cystic (perfectly round-shaped with a large lumen and thin cell border), intermediate (round-shaped with a large lumen but a thickening and uneven cell border) and mature (folded and budding) types of organoids through time (Figure $6 \mathrm{~A}$ and $6 \mathrm{D}$ ). We observed that organoid type proportion in FoxR1 cultures was overall significantly different from PaTa-1818x with a higher percentage of organoids from the cystic type and less mature organoids in FoxR1 cultures compared to PaTa-1818x $(p<0.001$, Figure 6D). Maximum organoid maturation was reached at the same time in PaTa-1818x compared to FoxR1 (d21.8) but the proportion of mature organoids was higher in PaTa-1818x compared to FoxR1 (8.33\% vs $12.94 \%$ ). Since cystic organoids are usually characterized by higher stemness and proliferation capabilities than mature, more differentiated organoids (Balak et al., 2019), these results are consistent with functional and molecular observations of our study suggesting that FoxR1 proliferation rate and stemness are higher than that of naive organoids.

Therefore, we used organoid forming efficiency as a largely accepted functional assay to measure stemness properties (Fujii and Sato, 2020) in FoxR1 compared to PaTa-1818x organoids. Organoid forming capacity of FoxR1 cells was significantly higher than that of PaTa-1818x naive cells ( 0.15 vs $0.085, p<0.001$, Figure $6 \mathrm{C}$ ).

Consequently, we analyzed the expression profiles of two pluripotency genes over 7 days of organoid culture after the beginning of their maturation (from d11). While OCT4 and NANOG expression progressively decreased, indicating rapid organoid differentiation usually associated with maturation and budding, from d11 to d18 in PaTa-1818x cultures (Figure 6E, left panel), we observed a much higher expression of OCT4 and an increased expression of NANOG on d17 in FoxR1 organoid cultures, leading to an opposite pluripotency signature when organoids are maintained in culture (Figure 6E, right panel). This is compatible with our hypothesis of a higher number of stem cell clusters in FoxR1 organoids compared to PaTa1818x leading to more complex mature 3D structures. Initial OCT4 expression (d11) was also slightly higher in FoxR1 than in PaTa-1818x. This may partially explain the delayed maturation and higher stemness functionality of FoxR1 organoids compared to naive PaTa1818x.

Altogether these results assessing the shape, size, number and expression profile of resistant compared to naive organoids indicate that repeated treatment with FOLFIRINOX 
imbalanced organoid cell hierarchy towards the immature cell compartments and increased stemness functional properties of PaTa-1818x PDAC organoids.

\section{Key signaling pathways are activated in FoxR1 compared to PaTa-1818x}

In order to better understand how FoxR1 organoids had acquired their resistance to FOLFIRINOX, we investigated the relative phosphorylation levels of 43 key kinase phosphorylation residues and two related total proteins ( $\beta$-catenin and HSP60) using a Human Phospho-Kinase Array (Figure 7A and supplemental table 1) in treated and untreated FoxR1 and PaTa-1818x organoids. The results are summarized in Figure 7D.

First, we compared FoxR1 organoids to PaTa-1818x (Supplemental table 1, first column). We observed an increased phosphorylation of the phosphoprotein "with no lysine [K]" WNK1 (1.213-fold) and an increased expression of $\beta$-catenin (1.246-fold) and HSP60 (1.324-fold). Although marginally significant in the array, these results were confirmed by western blotting in independent experiments (Figure 7B-C).

$\beta$-catenin accumulation in FoxR1 compared to $\mathrm{PaTa}-1818 \mathrm{x}$ is perfectly consistent with higher stemness functions that we observed in this study in resistant compared to naive organoids. FoxR1 organoids would therefore mimic a constant activation of the WNT pathway.

HSP60 is a mitochondrial chaperone that plays a key role in protein assembly and homeostasis. It has been recently suggested that targeting aberrant HSP60/OXPHOS/Erk1/2 phosphorylation axis through the use of drugs such as metformin may be beneficial to circumvent pancreatic cancer cell growth (Zhou et al., 2018). The role of HSP60 as an actor of PDAC chemoresistance to FOLFIRINOX will have to be confirmed in further studies. If so, such therapeutic strategy may be of even greater interest than previously suggested.

WNK kinases have increasingly gained interest in cancer progression these past few years, especially because of their drugability (Zhang et al., 2016). Interestingly, WNK1 phosphorylation is mediated by insulin-like growth factor (IGF)-1. It is associated with increases of phospholipase C- $\beta$ signaling, angiogenesis, and cell migration (Tsuboi et al., 2016). WNK1 phosphorylation may be an interesting path in order to understand and counteract pancreatic cancer resistance to FOLFIRINOX.

To analyze how FoxR1 respond to a new challenge of FOLFIRINOX compared to naive PaTa-1818x, we calculated phosphorylation ratios between FOLFIRINOX-retreated and basal FoxR1 organoids (Supplemental Table 1, column 3) and compared it to the ratios obtained for treated vs untreated PaTa-1818x (Supplemental Table 1, column 2) organoids. Our results show that ERK1/2 phosphorylation is significantly reduced in PaTa-1818x in response to FOLFIRINOX treatment (0.599-fold, Figure 7B-C). This reduction of phosphorylation between treated and untreated was not observed in FoxR1 organoids both 
in our array and western blotting experiments. Since ERK1/2 phosphorylation is associated with cell proliferation, these data are consistent with the greater cytostatic effect of FOLFIRINOX observed in PaTa-1818x compared to FoxR1 organoids. This also fits with significantly higher ERK1/2 phosphorylation observed in FOLFIRINOX-treated FoxR1 vs PaTa-1818x organoids (2.445- vs 0.599-fold, Figure 7B-C).

Surprisingly, we observed similar decreased in p53 phosphorylation on both S392 and S46 sites in both naive PaTa-1818x and resistant FoxR1 organoids. P53 phosphorylation on S46 residues are associated with p53-mediated induction of pro-apoptotic genes (Toledo et al., 2006; Taira et al., 2007), while $\$ 392$ phosphorylation is associated with p53 mitochondrial translocation and transcription-independent apoptosis (Castrogiovanni et al., 2018). However, Caspase and PARP cleavage in PaTa-1818x organoids after treatment clearly reflected apoptotic signals. These data may indicate that, at least in PaTa-1818x, other signaling pathways may be responsible for FOLFIRINOX-induced apoptosis.

The comparison of phosphorylation levels between FOLFIRINOX-treated PaTa-1818x and untreated FoxR1 (Supplemental Table 1, column 5) also gave us an insight into the signaling pathways that are activated along the successive cycles of FOLFIRINOX treatment and hereafter the acquisition of chemoresistance, as opposed to the effect of the first dose of chemotherapy.

Although marginally significant, AMPK $\alpha 1$ phosphorylation was weakly increased (1.313fold) in FoxR1 compared to FOLFIRINOX-treated PaTa-1818x organoids, in the array experiment (supplemental table 1). AMPK $\alpha 1$ plays a key role in cell metabolism. As a sensor of intracellular ATP reduction, AMPK activates energy-producing pathways and inhibits protein, carbohydrate and lipid biosynthesis and consequently cell proliferation (Faubert et al., 2013). Treatment with chemotherapy can affect tumor metabolism, as it has been shown for gemcitabine in PDAC through hypoxia-mediated signals (Shukla et al., 2017) or through endoplasmic reticulum stress (Tadros et al., 2017). However, little is known about metabolic alterations activated in FOLFIRINOX-treated pancreatic cancer cells and how it enhances PDAC chemoresistance. Our preliminary results suggest that FoxR1 organoids harbor higher basal respiration levels than naive organoids (data not shown). Since several identified signaling pathways and ROS accumulation in PDO evoke a metabolic switch, further investigations in this direction using this new model will surely bring insights into the metabolic mechanisms of chemoresistance in PDAC.

P70S6 kinase is a downstream actor of the AKT/mTOR signaling, a pathway that has already been identified as a key mechanism of pancreatic cancer resistance to gemcitabine (El Amrani et al., 2019). A weak increase of its phosphorylation was observed in our array experiment (1.209-fold) and was associated with an increased phosphorylation of AKT that was observed by western blotting between untreated FoxR1 and treated PaTa-1818x (2.35- 
fold, $p<0.05)$. Additionally, P70S6 kinase has been shown to have an anti-apoptotic activity through inactivation of the BCL2-associated agonist of cell death BAD (Harada et al., 2001). Hence, in our model, acquisition of resistance to apoptosis following FOLFIRINOX treatment may at least partly be due to activation of this kinase. More interestingly, phosphorylation of P70S6 is stimulated by amino acids in the presence of insulin and is therefore a way for the exocrine pancreas to sense amino acid availability in the microenvironment (Shah et al., 2001; Xu et al, 2001).

Altogether, these data could suggest an altered response of the insulin signature type which will have to be further explored and validated.

Finally, FAK phosphorylation was increased by 1.278-fold in FoxR1 compared to FOLFIRINOX-treated PaTa-1818x organoids. This was confirmed by western blotting in independent experiments (Figure 7B-C), suggesting that it could be implicated in the process of acquisition of resistance. FAK is largely involved in interactions of pancreatic cancer cells with their microenvironment. In a recent study, phosphorylated FAK has been associated with ITGB7 expression in pancreatic cancer cell lines (Sun et al., 2020). Hence, FAK hyperphosphorylation in our study in FoxR1 organoids compared to treated PaTa-1818x (14.98-fold) is associated with the overexpression of ITGB7 transcripts observed during acquisition of resistance to FOLFIRINOX. The FAK/ITGB7 may therefore be an interesting target to re-sensitize pancreatic cancer cells to these drugs.

To our knowledge, this is the first description of a rapid protocol for an in vitro model of acquired resistance to FOLFIRINOX in PDAC that can easily be adopted for research. Its relevance is illustrated by the fact that it reproduces clinical observations following PDAC treatment with FOLFIRINOX. Indeed, initial response to treatment is observed during the first three cycles of patient treatment (Conroy et al., 2018). Subsequently, since FoxR organoids slowly recover and re-enter the cell cycle, they rapidly mimic acquired chemoresistance and cancer relapse. The first insight into the mechanisms associated with acquired resistance that we observed are consistent with known pathways involved in sustained proliferation, anti-apoptotic signaling and stemness. Moreover, this study provides sequential information on acquired chemoresistance.

Unlike other studies aiming at modeling resistance by applying a constant chemotherapeutic pressure, we chose to alternate $72 \mathrm{~h}$ treatment phases with 4 days free of treatment. First, this permitted the use of concentrations of the three drugs that are close to physiological doses received by the tumor in vivo but that are too cytotoxic to be continuously used in vitro. Secondly, this allowed us to unravel the delayed effect of FOLFIRINOX treatment on cancer cells since highest cell mortality occurs several days after treatment 
arrest. Additionally, discontinued treatment more genuinely reflects the FOLFIRINOX protocol received by PDAC patients.

Relevant resistance models are mandatory to understand molecular mechanisms of chemoresistance while PDAC patient management still renders the comparison between the same tumors before and after treatment very arduous. This first new PDAC organoid model resistant to FOLFIRINOX represents an important step towards new opportunities to identify tomorrow's therapeutic strategies for pancreatic cancer. Indeed, comprehensive FOLFIRINOX-resistant PDAC organoid biobanks are now a reachable goal and will surely be dissected in the future using transcriptomic and epigenetic approaches to decipher further the mechanisms of chemoresistance. 


\section{Online supplemental Material}

\section{Supplemental Movies:}

Organoids were placed under an inverted Leica DMi8 microscope (Wetzlar, Germany) equipped with an incubation chamber for constant temperature and $\mathrm{CO}_{2}$ concentration (Okolab, $37^{\circ} \mathrm{C}$ and $5 \%$, respectively). Images were acquired every $4 \mathrm{~h}$ using a Hamamatsu Orca-Flash 4.0 LT camera (Hamamatsu city, Japan) and a 10x/0.3 objective. Live imaging acquisition was performed with Leica Application Suite Software for a total of 13 days. Time is indicated in the top left corner. Scale bar: $100 \mu \mathrm{m}$.

1: Time lapse imaging of PaTa-1818x organoids treated with FOLFIRINOX. Imaging of organoids that were treated with FOLFIRINOX from the beginning of day 1 through the beginning of day 4 .

Supplemental Movie 2: Time lapse imaging of FoxR1 organoids treated with FOLFIRINOX. Imaging of organoids that were treated with FOLFIRINOX from the beginning of day 1 through the beginning of day 4 .

\section{Supplemental Movie 3: Time lapse imaging of untreated PaTa-1818x organoids.}

\section{Supplemental Movie 4: Time lapse imaging of untreated FoxR1 organoids.}

Supplemental Figure 1: Acquisition of FOLFIRINOX resistant organoids. (A) Cell cycle analysis of organoid cells following FOLFIRINOX treatment. The cells were harvested from treated or control untreated organoids in their late phase of growth, stained with propidium iodide and anti-Ki67 antibody and DNA content was quantified by flow cytometry. Donut histograms show the percentage of cells in the SubG1, G0/G1, G1, S and G2/M phase of the cell cycle obtained after FACS analysis in control (Ctrl) untreated organoids or right before and after each cycle ( $\mathrm{C} 1$ to $\mathrm{C} 6)$ of FOLFIRINOX treatment. Histograms on the side reflect $\mathrm{G} 0$ vs. $\mathrm{G} 1$ proportions in the G0/G1 population for Ctrl and Post $\mathrm{C} 6$ conditions. For each sample 10,000 cells were acquired. Each experiment was repeated three times and values are representative of three replicates. (B) Orthotopic projections from bright field images of early growing PaTa-1818x organoids (10 days after seeding) before (pre C1) and after the $1^{\text {st }}$ (post $\mathrm{C} 1$ ) to the $6^{\text {th }}$ (post C6) cycle of $72 \mathrm{~h}$-treatment with FOLFIRINOX. The scale bar represents $500 \mu \mathrm{m}$ and is accurate for all images. (C) Cell cycle analysis of cells harvested from organoids in their early phase of growth following FOLFIRINOX treatment. 
(D) Representative flow cytometry histograms showing quantitative measurement of cell cycle phases from organoids in their early phase of growth.

Supplemental Figure 2: Gene expression analysis along the acquisition of resistance to FOLFIRINOX. Gene expression analysis of $c-m y c$ and CCNE1 proliferation genes, ABCG2, MRP1, MRP4 and MRP5 ABC transporters, LGR5, OCT4 and NANOG pluripotency genes, ITGB7 and S100A4 markers of pancreatic aggressiveness by RT-qPCR in treated organoids compared to naive organoids (expression arbitrary set to 1) before and after each cycle of FOLFIRINOX treatment (Cycle 1 to 6: C1 to C6). Data were obtained from three biological replicates from organoids in their late phase of growth (A, 20 days after cell seeding) or organoids in their early phase of growth (B, 10 days after seeding). (C) Mean ratio of mitochondrial superoxyde produced by organoids treated with FOLFIRINOX in their early phase of growth.

Supplemental Figure 3: PaTa-1818x and FoxR1 organoid growth ratios. Live organoid areas were measured using the Intellisis ZEN software (Zeiss). Average areas were calculated from more than 100 organoids obtained from six independent time-lapse images. Averages for each time frame were normalized with the average area of organoids from the first time frame of each organoid population arbitrary set to 1. Bars represent the S.E.M. Orange horizontal line represent time of treatment.

\section{Supplemental Table 1: Semi-quantitative analysis of the phospho-kinase array} experiment. The table shows the fold changes of 43 key kinase phosphorylation sites and two related total proteins with different comparisons. Phosphorylation sites showing foldchanges $<0.7$ were considered as inhibited (in green) and those $>1.4$ were considered activated (in red) as indicated in the table legend. Highlighted rectangles indicate the most relevant changes. Empty dashed red or green rectangles indicate marginally significant changes that are discussed for their relevance in chemoresistance. 


\section{Materials and Methods}

\section{Patient information}

This study was approved by the Scientific Committee of the tumour bank of Lille and the Department of Pathology of the Lille University Hospital. The patient, an 85-year-old male, had signed an informed consent. Distal pancreatectomy was carried out for a moderately differentiated pancreatic ductal adenocarcinoma in the Department of Digestive Surgery and Transplantation of the Lille University Hospital. The resected tumor was histologically diagnosed as PT2N1M0. The patient had not been treated with neoadjuvant chemotherapy.

\section{Primary organoid culture}

The tumour sample $\left(125 \mathrm{~mm}^{3}\right)$ was cut into small pieces $\left(<3 \mathrm{~mm}^{3}\right)$ and dissociated into small clusters and single cells in gentleMACS ${ }^{\mathrm{TM}} \mathrm{C}$ Tubes (Miltenyi Biotec) containing $3 \mathrm{~mL}$ of Advanced DMEM/F12 (ADF, Invitrogen \#11320-082) complemented with $6 U$ dispase (BD Biosciences, \#354235) and $2500 \mathrm{U}$ of collagenase IV (Sigma-Aldrich C1889). Two incubations at $37^{\circ} \mathrm{C}$ for $1 \mathrm{~h}$ under slow rotation were intersected with mechanical disruption using the human tumour program of the gentleMACS ${ }^{\text {TM }}$ Dissociator (Miltenyi Biotec). Cells and clusters were centrifuged, resuspended in ADF and filtered through a $70-\mu \mathrm{m}$ cell strainer. After centrifugation, cells were resuspended in Matrigel (Corning \#356231) and seeded in $40 \mu \mathrm{L}$ domes in the wells of a 24 -well plate. After Matrigel solidification, domes were covered with complete pancreatic tumor organoid medium (ADF medium supplemented with Glutamax [1×, Invitrogen \#35050-061], HEPES [1x, Sigma-Aldrich \#83264-100ML-F], B$27^{\circledR}$ Supplement Minus Vitamin A (Invitrogen \#12587-010), N2 [1×, Invitrogen \#17502-048], $\mathrm{N}$-acetyl-L-cysteine [1 mM, Sigma-Aldrich], Wnt3a/RSPO1/Noggin-conditioned medium [50\% $\mathrm{v} / \mathrm{v}$ ], epidermal growth factor [EGF, $50 \mathrm{ng} / \mathrm{ml}$, Peprotech AF-100-15], fibroblast growth factor 10 [FGF10, 100 ng/ml, Preprotech \#100-26], ALK-5 and Smad signaling inhibitor A83-01 [0.5 $\mu \mathrm{M}$, Tocris \#2939], Nicotinamide [10 mM, Sigma-Aldrich \#N0636], Gastrin [10 nM, SigmaAldrich \#G9145] and ROCK Inhibitor Y-27632 dihydrochloride [10 $\mu \mathrm{M}$, Tocris \#1254]) as recommended in Boj et al., 2015. Resulting PDAC organoids were named PaTa-1818x (PAncreatic TAil). Culture was then carried out according to (Boj et al., 2015). Complete medium without ROCK inhibitor was then added every two days and organoids were passaged through mechanical disruption every two weeks.

\section{Histopathological Evaluation}

Pancreatic cancer samples were immediately transmitted to the Department of Pathology after surgical resection and fixed in formalin $4 \%$ and then paraffin-embedded. Alternatively, 
tumor-derived organoids were fixed in formalin 4\%, gently harvested in prewarmed Thermo Scientific ${ }^{\mathrm{TM}}$ Richard-Allan Scientific HistoGel ${ }^{\mathrm{TM}}$ Specimen Processing Gel with a cell scraper and then paraffin-embedded. Microscopic examination was performed on 3- $\mu$ m slides stained either with hematoxylin and eosin (HE) or alcian blue. Examination was performed at the pathology department (CHU Lille) by F.R.

\section{Mutation analysis}

The NucleoSpin Tissue kit (Macherey-Nagel) was used for extraction of genomic DNA from organoids according to the manufacturer's instructions for DNA isolation from tissues. For Next Generation Sequencing (NGS), the custom-made panel was designed using the lon AmpliSeq Designer Software (ThermoFisher Scientific ${ }^{\circledR}$ ) to identify somatic mutations in ACVR1, AKT1, ALK, BRAF, CTNNB1, EGFR, ERBB2, ERBB4, FGFR1, FGFR2, FGFR3, GNA11, GNAQ, GNAS, H3F3A, H3F3B, HIST1H3B, HIST1H3C, HRAS, IDH1, IDH2, KRAS, KIT, MAP2K1, MET, NRAS, PDGFRA, PIK3CA, PTEN, PTPN11, SMAD4, and TP53 genes (Descarpentries et al., 2018). PaTa-1818x and FoxR1 DNA samples (10 ng) were used as template to prepare the library according to the manufacturer's instructions. Ampliseq libraries were prepared using the lon Ampliseq Library Kit 2.0 (ThermoFisher Scientific ${ }^{\circledR}$ ) and barcoded using the Ion Xpress Barcode Adapters Kit (ThermoFisher Scientific ${ }^{\circledR}$ ). Quality control and quantification of amplified libraries were performed on the 2200 TapeStation (Agilent Technologies ${ }^{\circledR}$, Santa Clara, CA, USA) using the High Sensitivity D1000 ScreenTape assay. Amplified libraries were normalized and pooled. Pooled libraries were clonally amplified on Ion Sphere Particles (ISPs) by Emulsion PCR. Amplification, ISPs enrichment and chip loading were performed on lon Chef Instrument with the lon 540 Kit-Chef or the Ion PI Hi-Q Chef Kit (ThermoFisher Scientific ${ }^{\circledR}$ ). Template ISPs were sequenced on the lon S5 $X L$ Sequencer (ThermoFisher Scientific ${ }^{\circledR}$ ). Each run included a positive control, a negative control and a non-template control to validate quality of the assay. Data were analyzed using the Torrent Suite Software v.5.2.2 (ThermoFisher Scientific ${ }^{\circledR}$ ). Variant calling was performed with optimized settings. Variants were annotated with Variant Effect Predictor. The lower limit of detection for NGS assays is $5 \%$ of mutated DNA. For a wild-type a minimum call of $300 \mathrm{X}$ is required.

\section{Organoid treatment with FOLFIRINOX and IC50 measurement}

Organoids were harvested and dissociated into single cells by digesting with TrypsinEDTA ( $0.25 \%$, Invitrogen). The dissociated cells were counted before resuspension into Matrigel domes.

For the process of acquisition of resistance, organoids were first treated with FOLFIRINOX (4 $\mu \mathrm{M}$ of 5-FU (Sigma-Aldrich \#F6627), $0.5 \mu \mathrm{M}$ of oxaliplatin (Sigma-Aldrich 
\#O9512) and $12.5 \mathrm{nM}$ of SN-38 (Sigma-Aldrich \#H0165) for 72h) either 10 (organoids in early phase of growth) or 20 (late phase of growth) days after seeding. Medium was then entirely removed and replaced with fresh complete medium for four days before the next cycle of treatment.

For organoid forming assays, $5 \times 10^{3}$ single cells per $50 \mu$ l Matrigel dome were plated in 24-well plates.

For time-lapse imaging, dissociated cells were seeded in $20 \mu \mathrm{L}$ Matrigel domes (6 per well of a 6-well plate). Organoids were treated with FOLFIRINOX for $72 \mathrm{~h}$. Medium was then entirely removed and replaced by fresh complete medium.

For FOLFIRINOX IC50 measurement, 96-well plates were precoated with Matrigel diluted $1: 2$ in complete culture medium. Dissociated cells $\left(1 \times 10^{3}\right.$ per well) were resuspended in 200 $\mu \mathrm{L}$ medium supplemented with $2 \%$ Matrigel and $10 \mu \mathrm{M}$ ROCK Inhibitor Y-27632 dihydrochloride (Tocris \#1254). On the day of treatment, $100 \mu \mathrm{L}$ of culture medium was removed and replaced by $100 \mu \mathrm{L}$ of complete medium containing serial dilutions of FOLFIRINOX from $50 \mathrm{X}$ to $0.02 \mathrm{X}$ (8 wells per condition), $1 \mathrm{X}$ corresponding to $4 \mu \mathrm{M}$ of 5 -FU, $0,5 \mu \mathrm{M}$ of oxaliplatin and $12,5 \mathrm{nM}$ of $\mathrm{SN}-38$. After $72 \mathrm{~h}$, the totality of the medium was removed and replaced by $200 \mu \mathrm{L}$ of complete medium. $48 \mathrm{~h}$ after the end of the treatment, ATP levels were quantified using CellTiter-Glo 3D cell viability assay (Promega) according to the manufacturer's instruction and luminescence was measured using Centro XS3 LB 960 (Berthold technologies) with Miko Win 2000 software. Results were normalized to vehicle $($ DMSO $=100 \%)$.

\section{Organoid imaging}

Organoids were placed under an inverted Leica DMi8 microscope (Wetzlar, Germany) equipped with an incubation chamber for constant temperature and $\mathrm{CO}_{2}$ concentration (Okolab, $37^{\circ} \mathrm{C}$ and $5 \%$, respectively). Images were acquired every $4 \mathrm{~h}$ using a Hamamatsu Orca-Flash 4.0 LT camera (Hamamatsu city, Japan) and a 10x/0.3 objective. Live imaging acquisition was performed with Leica Application Suite Software from three regions per well for a total of 13 days.

Alternatively, bright field images of organoids were obtained with an inverted Olympus cellVivo microscope (Tokyo, Japan) using a Zyla 5.5 sCMOS camera and a x10/0.3 objective and the CellSens software.

For the process of acquisition of resistance, bright-field images of entire Matrigel domes were obtained through tiling and z-stack experiments performed using the Cell Discoverer 7 (Zeiss) and a 5x/0.35 objective. Orthotopic projection was then obtain from the acquisition through averaging of all stacks with the maximum thickness using the ZEN software. 


\section{Surface measurement and organoid type recognition}

ZEISS Intellisis of ZEN software (Jena, Germany) was trained to automatically recognize and measure the area of all types of organoids and subsequently to classify objects corresponding to cystic, intermediate and mature organoids. This module is an advanced tool which utilizes established open-source machine learning algorithms powered by Python Dask, Scikit-Learn and Tensorflow 2 and deep learning to segment pixels. It allows the construction of a classifier from a range of different features extracted from all types of images, including local and non-local grey scale, gradient and texture.

\section{Flow cytometry}

Cells were labelled using functional probes for mitochondrial oxydative stress with Mitosox Red, viability with Sytox Blue and cell cycle with Propidium lodide and Ki67 antibody (Table 2).

For oxidative stress analysis, organoids from six culture domes were trypsinized, harvested from treated or untreated organoids as controls and incubated in $600 \mu \mathrm{L}$ of DMEM medium supplemented with $0.5 \mathrm{mM}$ EDTA. The Mitosox Red probe was added for 30 minutes at $37^{\circ} \mathrm{C}$. First acquisition was performed on a minimum of 5000 cells. Sytox blue was then added to each tube for 10 minutes on ice. A new acquisition was performed on a minimum of 10000 cells. Analysis was done on cells selected for their viability.

For cell cycle analysis, cells were trypsinized, harvested from treated or untreated organoids as controls and fixed with cold $70 \%$ ethanol for 30 minutes at $4^{\circ} \mathrm{C}$, washed with $1 \mathrm{X}$ PBS before resuspension with $1 \mathrm{X}$ PBS and treatment with RNase $\mathrm{A}$ at a final concentration of $2 \mu \mathrm{g} / \mathrm{mL}$ for 15 minutes at room temperature. The cells were then incubated with the primary anti-Ki67 antibody for 30 minutes on ice and the secondary anti-rabbit conjugated antibody for another 30 minutes on ice. Staining with Propidium lodide-Triton X100 $(0.25 \mu \mathrm{g} / \mathrm{mL}-0.1 \%)$ was then performed at room temperature and protected from light for 30 minutes. Analysis were done in order to distinguish the different phases of the cell cycle in addition to the apoptosis phase (subG1) and polyploid cells on single cell selection. Flow analysis was performed using the LSR Fortessa X20 Cell Analyzer (BD Biosciences Inc.). The analysis of raw data was performed using the KALUZA Analysis software (v2.1, Beckman Coulter Inc.).

\section{Quantitative reverse-transcriptase polymerase chain reaction}

Total RNA from pancreatic tumour organoids were extracted using the NucleoSpin RNA II kit (Macherey Nagel) following manufacturer's instructions. Complementary DNA was synthesized as previously described with Oligod(T) primers (Vincent et al., 2008). Quantitative polymerase chain reaction (qPCR) was performed using the SsoFast EvaGreen 
Supermix using the CFX96 REAL-Time PCR Detection System on a C1000 Thermal Cycler (Bio-Rad). Primer information is listed in Table 1. Relative gene expression levels $\left(2^{-\Delta \Delta C t}\right)$ were normalized to the RPLPO housekeeping gene.

\section{Western-Blotting}

FoxR1 and PaTa-1818x were treated with FOLFIRINOX for $72 \mathrm{~h}$ in 6 -well plates (6 Matrigel domes per well). Every $24 \mathrm{~h}$, organoids were harvested by centrifugation, rinsed with ice cold $1 \mathrm{X}$ phosphate buffer saline (PBS) before being resuspended in lysis buffer $(50 \mathrm{mM}$ Tris-HCL pH 8.0 containing $150 \mathrm{mM} \mathrm{NaCl}, 1 \%$ (w/v) NP40, 0.5\% (w/v) sodium deoxycholate and $0.1 \%$ SDS supplemented with Complete Protease Inhibitor Cocktail (Tablets, SigmaAldrich/Roche) and stored at $-80^{\circ} \mathrm{C}$ until use for western blotting. Before use, tubes containing cell lysates were thawed on ice, scratched on a metal rack and centrifuged at 14 $000 \times g$ at $4^{\circ} \mathrm{C}$ for 10 minutes. Supernatants were collected and protein concentrations were measured using the BCA Protein Assay Kit (Thermo Scientific ${ }^{\mathrm{TM}}$ Pierce). Protein extracts (20 $\mu \mathrm{g}$ ) were heated at $70^{\circ} \mathrm{C}$ for 10 minutes and were loaded into precast $4-12 \%$ NuPAGE BisTris gels (Thermo Fisher Scientific) followed by a transfer onto a nitrocellulose membrane (GE Healthcare Life sciences). After blocking with $5 \%(\mathrm{w} / \mathrm{v})$ dry milk in TBS (Tris-buffered saline) with $0.05 \%(\mathrm{v} / \mathrm{v})$ Tween20 (TBST)), membranes were probed with specific primaries antibodies overnight at $4^{\circ} \mathrm{C}$. The following antibodies were used: mouse anti- $\beta$-actin (Invitrogen \#31430; 1:5000), rabbit anti-Caspase-3 (Cell Signaling Technology \#9662; 1:400), rabbit anti-Cleaved Caspase-3 (Asp175, Cell Signaling Technology \#9664; 1:250), rabbit anti-PARP1 (Cell Signaling Technology \#9542; 1:1000), rabbit anti-H2AX (phosphoHistone H2A.X (ser139, Cell Signaling Technology \#9718; 1:1000), rabbit anti-ITGB7 (Mybiosource \#mbs127722; 1:500), rabbit anti-WNK1 (\#4979, Cell Signaling, 1:500), rabbit anti-phospho-WNK1 (T60, \#4946, Cell Signaling, 1:500), rabbit anti-FAK (\#13009, Cell Signaling, 1:500), rabbit anti-phospho-FAK (Y397, \#8556, Cell Signaling, 1:500), rabbit antiAKT (\#4691, Cell Signaling, 1:500), rabbit anti-phospho-AKT (T308, \#13038, Cell Signaling, 1:500), rabbit anti-ERK1/2 (\#9102, Cell Signaling, 1:500), rabbit anti-phospho-ERK1/2 (T802/Y204, \#4370, Cell Signaling, 1:500), rabbit anti-phospho- $\beta$-catenin (S675, \#4176, Cell Signaling, 1:500), rabbit anti-HSP60 (\#4869, Cell Signaling, 1:500), rabbit anti- $\beta$-catenin (\#8480, Cell Signaling, 1:500). Membranes were then incubated $2 \mathrm{~h}$ at room temperature with horseradish peroxidase-secondary antibodies (Sigma- Aldrich) and revealed using West Pico chemiluminescent substrate (Perbio, Villebon/Yvette, France) and the ImageQuant LAS 4000 imager (GE Healthcare Life Sciences). Pixel densitometry from ImageJ software was used to quantify proteins.

\section{Proteome Profiler Array}


For Proteome profiler array, $250 \mu \mathrm{g}$ of total protein extract from each condition were used to run on membranes of the Human Phospho-Kinase Array Kit (Proteome Profiler ${ }^{T M}, R \& D$ systems) according to the manufacturer's instructions. Chemiluminescence was detected using Image Quant LAS 4000 imager (GE Healthcare Life Sciences) and signal was carried out with the Image Quant TL software (GE Healthcare Life Sciences).

\section{Statistical analysis}

All values are mean values \pm S.E.M. unless specified otherwise. When indicated, data were analysed using GraphPad Prism 4 software with differences $p<0.05$ considered significant and represented with *. When $p$ values were below 0.01 and 0.001 , symbols ** and ${ }^{* * *}$ were used, respectively. 


\section{Figure legends}

Figure 1: Strategy to develop an in vitro model of pancreatic ductal adenocarcinoma chemoresistance. (A-L) Representative results of histological examination of the original primary PDAC (A-C, G-I) and derived tumor organoids PaTa1818x (D-F, J-L). (B-C) Higher magnifications from (A). (F) Higher magnification from (D). Typical cubic cells, designated by blue arrows, are organized in tubular structures as revealed by Hematoxylin-Eosin staining (A-F). Signet-ring cells (black arrows) and mucinproducing cells are revealed by alcian blue staining (G-L). Scale bars represent $100 \mu \mathrm{m}$. (M) Scheme representing bicompartmental diffusion of oxaliplatin and irinotecan/SN-38 drugs in the bloodstream (black cylinder) and tumour tissue (black rectangle) over time. Plasmatic concentrations are characterized by curves with first a highly negative slope due to distribution of the drug into the tissues along with elimination (in purple). Then, plasma and tissue concentrations reach equilibrium. This phase is followed by a decrease of the slope, which represents pure elimination (in green). (N) Scheme showing the protocol of PaTa1818x treatment used to generate FOLFIRINOX-resistant organoids FoxR. Cycles of one week (day (d) 0 to $\mathrm{d} 7$ ) constituted of $72 \mathrm{~h}$ of treatment (orange squares) followed by 4 days without treatment (white squares) were repeated six times. Organoids were harvested at the beginning (d1, d7, d14, d21, d28 and d35) and at the end (d3, d10, d17, d24, d31 and d38) of each FOLFIRINOX treatment in order to compare cell cycle distribution, gene expression and mitochondrial superoxyde production in treated vs untreated organoids (vertical black arrows). Representative bright-field microscopy images of mature PaTa-1818x (left and up right images) and FoxR1 (bottom right image) organoids are shown. Scale bars represent $100 \mu \mathrm{m}$.

Figure 2: Acquisition of FOLFIRINOX resistant organoids. (A) Orthotopic projections of bright field images of PaTa-1818x organoids after the $2^{\text {nd }}$ (post C2) to the $6^{\text {th }}$ (post C6) cycle of $72 \mathrm{~h}$-treatment with FOLFIRINOX. Scale bars represent $500 \mu \mathrm{m}$. The black arrows show tumor cells invading out of a mature organoid. Areas indicated by a black rectangle are shown at higher magnification in outer images. (B) Cell cycle analysis of organoid cells following FOLFIRINOX treatment. The cells were stained with propidium iodide and anti-Ki67 antibody (proliferating cells) and DNA content was quantified by flow cytometry. Donut histograms show the percentage of cells in the SubG1, G0/G1, G1, S and G2/M phase of the cell cycle obtained after FACS analysis in control (Ctrl) untreated organoids and after each cycle of FOLFIRINOX treatment. Lower donut histograms show the repartition in G0 (in grey) vs. G1 (in green) in Ctrl and post C6 conditions. For each sample 10,000 cells were 
acquired. Values are representative of three independent replicates. (C) Representative flow cytometry histograms showing quantitative measurement of cell cycle phases.

Figure 3: Gene expression changes along the acquisition of resistance to FOLFIRINOX (A) Analysis of expression of $c$-myc and CCNE1 proliferation genes, ABCG2, MRP1, MRP4 and MRP5 ABC transporters, LGR5, OCT4 and NANOG pluripotency genes, ITGB7 and S100A4 markers of pancreatic aggressiveness by RT-qPCR in treated organoids compared to naive organoids expression arbitrary set to 1 before the beginning of the first treatment ( $\mathrm{d} 0$, Cycle 1: $\mathrm{C} 1$ ) and after each of the six cycles of FOLFIRINOX treatment (post $\mathrm{C} 1$ to post $\mathrm{C6}$ ). Data were obtained from three biological replicates and expressed as mean \pm S.E.M. Results of unpaired $t$-test are represented with * for $p<0.05$ and ${ }^{* *}$ for $p<0.01$, respectively. (B) Analysis of ITGB7 protein expression by western blotting. Densitometric quantifications were carried out using the GelAnalyzer software. (C) Analysis of mitochondrial superoxyde production. The cells were harvested and stained with MitoSOX Red Mitochondrial Superoxide Indicator before and after each cycle of treatment (C1 to C6). Fluorescence was quantified by flow cytometry. For each sample 10,000 cells were acquired. Values are the mean of three independent replicates \pm SEM. Unpaired $t$-test with * for $p<0.05$.

Figure 4: FoxR1 organoids are more resistant to FOLFIRINOX than naive PaTa1818x. (A) Bright field images of PaTa-1818x (upper panel) and FoxR1 (lower panel) organoids treated with FOLFIRINOX extracted from Supplemental movies 1 and 2, respectively. Organoids were treated for $72 \mathrm{~h}$ (orange horizontal line) from the beginning of day (d) 1 to the beginning of $\mathrm{d} 4$ as depicted in the upper scheme (filled squares). Double black arrows represent the time points at which comparisons were made between treated PaTa-1818x and FoxR1. Cell mortality and consequent loss of organoid border integrity are indicated by white arrows. Scale bars: $200 \mu \mathrm{m}$. (B) Bar graph representing organoid viability during (orange horizontal line) and after a $72 \mathrm{~h}$ treatment with FOLFIRINOX. Organoid loss of structure linked to cell mortality was counted manually from at least six images at the beginning of the treatment ( $d 1$ ), and on $d 4, d 5, d 6, d 10$ and d13. Bars represent the mean \pm standard deviation (sd). Statistical significance was evaluated using 2-way ANOVA for the time lapse followed by Bonferroni posttests for each time frame $\left({ }^{*}: p<0.05 ;{ }^{* *}: p<0.01\right)$. (C) Organoid growth curves. Live organoid areas were measured using the Intellisis ZEN software (Zeiss). Averages \pm S.E.M. were calculated from more than 100 organoids obtained from six independent time-lapse images. Statistical significance was evaluated using 2-way ANOVA for the time lapse followed by Bonferroni posttests for each time frame $\left({ }^{*}: p<0.05 ;{ }^{* *}\right.$ : $p<0.01 ;^{* * *}: p<0.001$; ns: not significant). (D) Graphical representation of the heterogeneity in size and number of live organoid areas measured using the Intellisis ZEN software (Zeiss) 
before, during and after FOLFIRINOX treatment. Blue dots represent each measured organoid from the PaTa-1818x population and orange dots represent FoxR1 organoids from six images acquired in one experiment that was repeated three times. Organoid areas (expressed as $\log \mu \mathrm{m}^{2}$ ) are presented as white boxplots, indicating medians and quartiles. The colored violin plots illustrate kernel probability density: the width of the shaded area represents the proportion of organoids of each size. Smooth conditional means for treated FoxR1 and PaTa-1818x along time are also illustrated using orange and blue lines, respectively.

Figure 5: FoxR organoids are more resistant to FOLFIRINOX-induced apoptosis than naive PaTa-1818x. (A) Scheme illustrating the experiment time points. Double black arrows represent the time points at which comparison was made between treated PaTa1818x and FoxR1. The asterisk indicates the time point used for IC50 measurement. (B) Analysis of Caspase 3 expression (FL: Full length) and cleavage, PARP-1 expression (FL) and cleavage, and Histone variant $\mathrm{H} 2 \mathrm{AX}$ phosphorylation $(\gamma \mathrm{H} 2 \mathrm{AX})$ by Western blotting in Pata-1818x (PaTa or P) and FoxR (or F) organoids treated (+) or not (-) with FOLFIRINOX for $72 \mathrm{~h}$. Densitometric quantification was carried out three independent biological replicates using the Gel Analyzer software and values of $\mathrm{d} 5$ are reported in the bar graphs below representative images. For $\gamma \mathrm{H} 2 \mathrm{AX}, \beta$-Actin was used as loading control. Results of unpaired $t$-test are represented with * for $p<0.05$. (C) Survival curves of organoids after exposure to serial dilutions of FOLFIRINOX from $50 \mathrm{X}$ to $0.02 \mathrm{X}$ for $72 \mathrm{~h}$. Bars represent the mean $\pm \mathrm{SD}$ of 8 replicates.

Figure 6: Growth and stemness characteristics of resistant FoxR1 compared to naive PaTa-1818x organoids. (A) Bright field images of PaTa-1818x and FoxR1 untreated organoids extracted from Supplemental movies 3 and 4, respectively. Time-lapse imaging was started 11 days (d11) after individual cell seeding in Matrigel ${ }^{\circledR}$ domes. Scale bar: 200 $\mu \mathrm{m}$. Arrows indicate cystic (perfectly round-shaped with a thin border) in white, intermediate (round-shaped with a thick border) in grey and mature (folded and budding) organoids in black. (B) PaTa-1818x and FoxR1 organoid growth curves. Organoid areas (in $\mu \mathrm{m}^{2}$ ) were automatically measured using the Intellisis ZEN software (Zeiss). Statistical significance was evaluated using 2-way ANOVA for the time lapse followed by Bonferroni posttests for each time frame $\left({ }^{*}: p<0.05 ;{ }^{* *}: p<0.01\right.$; ns: not significant). (C) Graph bars representing mean numbers of organoids formed from 5000 individual cells seeded in $50 \mu \mathrm{L}$ Matrigel domes. Error bars represent standard deviations. Statistical significance was evaluated using Student's $t$ test $\left.{ }^{* * *}: p<0.001\right)$. (D) Graph bars representing percentages of cystic (white), intermediate (grey) and mature (black) organoids through time in FoxR1 (right panel) compared to PaTa-1818x (left panel) organoid culture. Different types of organoids were 
identified using the Intellisis software (Zeiss). Statistical significance $(p)$ was evaluated using 2-way ANOVA and was <0.001. (E) Analysis of expression of OCT4 and NANOG pluripotency genes by RT-qPCR in resistant FoxR1 compared to naive Pata-1818x organoids expression arbitrary set to 1 , from day $(d) 11$ to $d 17$. Data were obtained from three independent experiments and expressed as mean \pm Standard Error of the Mean (S.E.M.). Results of unpaired $t$-test are represented with ** for $p<0.01$ and ns for non-significant, respectively.

Figure 7: Differential signaling profiles of FoxR1 compared to PaTa-1818x. A phospho-kinase array was run 48h after the end of a 72h-FOLFIRINOX treatment or on the same day in untreated organoids. (A) Images of the phospho-kinase array experiment from cell lysates corresponding to treated (FOLFIRINOX) or untreated FoxR1 and PaTa-1818x organoids. Differential phosphorylation signals are indicated with numbered dark blue rectangles (treated compared to untreated $\mathrm{PaTa}-1818 \mathrm{x}$ ), orange rectangles (untreated FoxR1 compared to untreated PaTa-1818x), orange discontinued rectangles (untreated FoxR1 compared to treated PaTa-1818x) or brown rectangles (treated FoxR1 compared to treated PaTa-1818x or untreated FoxR1). (B) Validation of protein expression changes identified by the phospho-kinase array experiment by Western blotting. Densitometric quantification was performed using Image $\mathrm{J}$ and are reported in the bar graphs as mean ratios (phosphorylated protein/total protein) from at least three independent experiments \pm Standard Error of the Mean (S.E.M.). HSP60 band intensities were normalized on $\beta$-actin. Results of unpaired $t$-test are represented with * for $p<0.05$. (C) Representative analysis of expression of HSP60 and phosphorylation status of WNK1 ( $p$-WNK1), FAK (p-FAK), $\beta$ Catenin ( $p$ - $\beta$-Catenin), AKT ( $p$-AKT) and ERK1/2 (p-ERK1/2) by Western blotting in Pata1818x (PaTa or P) and FoxR1 (F) organoids treated (+) or not (-) with FOLFIRINOX for 72h. (D) Scheme representing key signaling pathways modified in resistant organoids and identified using phospho-kinase array (in blue) and independently validated (in orange) with significantly altered phosphorylation rates (in red). 
Table 1: Sequences of the primers used for RT-qPCR

\begin{tabular}{|c|c|c|c|}
\hline \multirow{2}{*}{ Primer } & \multicolumn{2}{|c|}{ Sequence $5^{\prime} \rightarrow 3^{\prime}$} & \multirow{2}{*}{ Amplified gene } \\
\hline & forward & reverse & \\
\hline$A B C G 2$ & ACGAACGGATTAACAGGGTCA & CTCCAGACACACCACGGAT & $\begin{array}{l}\text { ATP Binding Cassette } \\
\text { Subfamily G Member } 2\end{array}$ \\
\hline CYCLIN E1 & ACTCAACGTGCAAGCCTCG & GCTCAAGAAAGTGCTGATCCC & $C C N E 1$ \\
\hline$C-M Y C$ & TCTGTGGAAAAGAGGCAGGCTCC & TGCTGATGTGTGGAGACGTG & MYC \\
\hline ITGB7 & ATGGTGGCTTTGCCAATGGT & GGACAGGTGAGGATTCCGC & Integrin Subunit Beta 7 \\
\hline S100A4 & CCACAAGTACTCGGGCAAAG & GTCCCTGTTGCTGTCCAAGT & $\begin{array}{c}\text { S100 Calcium Binding Protein } \\
\text { A4 }\end{array}$ \\
\hline$M R P 1$ & CTGACAAGCTAGACCATGAATGT & TCACACCAAGCCGGCGTCTTT & $\begin{array}{l}\text { Multidrug resistance-associated } \\
\text { protein } 1\end{array}$ \\
\hline MRP4 & GGATCCAAGAACTGATGAGTTAAT & TCACAGTGCTGTCTCGAAAATAG & $\begin{array}{l}\text { Multidrug resistance-associated } \\
\text { protein } 4\end{array}$ \\
\hline MRP5 & GCTGTTCAGTGGCACTGTCAG & TCAGCCTTGACAGCGACCTT & $\begin{array}{l}\text { Multidrug resistance-associated } \\
\text { protein } 5\end{array}$ \\
\hline NANOG & СCССАGССТTTАСТСТТССТА & CCAGGTTGAATTGTTCCAGGTC & NANOG1 \\
\hline OCT4 & GGGAGATTGATAACTGGTGTGTT & GTGTATATCCCAGGGTGATCCTC & POU5F1 \\
\hline LGR5 & GATGTTGCTCAGGGTGGACT & GGGAGCAGCTGACTGATGTT & $\begin{array}{c}\text { Leucine Rich Repeat } \\
\text { Containing G Protein-Coupled } \\
\text { Receptor } 5\end{array}$ \\
\hline RPLPO & GCAATGTTGCCAGTGTCTG & GCCTTGACCTTTTCAGCAA & $\begin{array}{c}\text { Ribosomal Protein Lateral Stalk } \\
\text { Subunit P0 }\end{array}$ \\
\hline
\end{tabular}

Table 2: Functional probes and antibodies used for flow cytometry

\begin{tabular}{|c|c|c|c|c|}
\hline \multicolumn{2}{|c|}{ viability } & $\begin{array}{c}\text { Mitochondrial } \\
\text { oxydative stress }\end{array}$ & Cell Cycle & \\
\hline Probe/antibody & Sytox blue & Mitosox Red & Prodidium iodide & Ki-67+Alexa488 \\
\hline Reference & $\begin{array}{c}\text { Thermofisher } \\
\text { S34857 }\end{array}$ & $\begin{array}{c}\text { Thermofisher } \\
\text { M36008 }\end{array}$ & $\begin{array}{c}\text { Sigma } \\
\text { P4864 }\end{array}$ & $\begin{array}{c}\text { Abcam ab15580+ } \\
\text { Thermofisher A-11008 }\end{array}$ \\
\hline $\mathbf{C}_{\mathbf{i}}$ & $1 \mathrm{mM}$ & $5 \mathrm{mM}$ & $1 \mathrm{mg} / \mathrm{ml}$ & - \\
\hline $\mathbf{C}_{\mathbf{f}}$ & $1 \mu \mathrm{M}$ & $2.5 \mu \mathrm{M}$ & $25 \mu \mathrm{gg} / \mathrm{ml}$ & $1 \mathrm{~g} \mathrm{PBS}$ \\
\hline Reaction buffer & $\begin{array}{c}\mathrm{DMEM}+ \\
\text { EDTA } 0.5 \mathrm{mM}\end{array}$ & $\begin{array}{c}\mathrm{DMEM}+ \\
\text { EDTA } 0.5 \mathrm{mM}\end{array}$ & $1 \mathrm{X} \mathrm{PBS}$ & $488 \mathrm{~nm}$ \\
\hline Laser excitation & $405 \mathrm{~nm}$ & $488 \mathrm{~nm}$ & $561 \mathrm{~nm}$ & $585 / 42$ \\
\hline
\end{tabular}

Ci: initial concentration ; Cf: final concentration 


\section{Author contribution}

Charles Poiraud initiated the concept and design of the research, wrote the article and analyzed and interpreted the data.

Elsa Hadj Bachir designed the research, carried out the experimental work, analyzed and interpreted the data and wrote the article.

Sonia Paget, Soumaya El Moghrabi, Louis-Bastien Weiswald, Laurent Poulain, Marie Vandepeutte César Beugniez and Belinda Duchêne carried out the experimental work.

Nicolas Stoup carried out signaling pathway experiments and analysis.

Nathalie Jouy analyzed flow cytometry data.

Antonino Bongiovanni and Meryem Tardivel provided tools for time-lapse microscopy and analyzed the data.

Chann Lagadec and Nicolas Jonckheere performed statistical analysis.

The OrgaRES consortium, Florence Renaud, Emmanuelle Leteurtre, Fabienne Escande and Stephanie Truant provided human samples for the establishment of patient-derived organoids and performed their histological and genetic characterization.

Pascal Pigny provided reagents and critical reading of the paper.

Isabelle Van Seuningen initiated the research on organoids.

Audrey Vincent initiated the concept and design of the research, wrote the article, analyzed and interpreted the data.

All authors have read and agreed to the submitted manuscript. 


\section{References}

Ahmed, F., Vyas, V., Cornfield, A., Goodin, S., Ravikumar, T.S. Rubin, E.H., and Gupta, E. (1999). In vitro activation of irinotecan to SN-38 by human liver and intestine. Anticancer Res, 2067-71.

Amours, D. D., Sallmann, F. R., Dixit, V. M., \& Poirier, G. G. (2001). Gain-of-function of poly(ADP-ribose) polymerase-1 upon cleavage by apoptotic proteases: implications for apoptosis. Journal of Cell Science 114, 3771-3778.

Amsterdam, A., Raanan, C., Schreiber, L., Polin, N., \& Givol, D. (2013). Biochemical and Biophysical Research Communications LGR5 and Nanog identify stem cell signature of pancreas beta cells which initiate pancreatic cancer. Biochemical and Biophysical Research Communications, 433(2), 157-162.

Andrikou, K., Santoni, M., Piva, F., Bittoni, A., Lanese, A., Pellei, C., ... Cascinu, S. (2015). Lgr5 expression, cancer stem cells and pancreatic cancer: results from biological and computational analyses. Future Oncology, 11, 1037-1045.

Baker, L. A., Clevers, H., Tuveson, D. A., Harbor, C. S., Pancreatic, L., Harbor, C. S., ... Cancer, K. (2017). Modeling pancreatic cancer with organoids. Trends Cancer, 2(4), 176-190.

Balak, J. R. A., Juksar, J., Carlotti, F., Nigro, A. Lo, \& Koning, E. J. P. De. (2019). Organoids from the Human Fetal and Adult Pancreas. Current Diabetes Reports, 19:160.

Begicevic, R., \& Falasca, M. (2017). ABC Transporters in Cancer Stem Cells: Beyond Chemoresistance. Int. J. Mol. Sci., 18, 2362.

Beumer, J., \& Clevers, H. (2016). Regulation and plasticity of intestinal stem cells during homeostasis and regeneration, 3639-3649.

Bleijs, M., Wetering, M., Clevers, H., \& Drost, J. (2019). Xenograft and organoid model systems in cancer research. The EMBO Journal, 38(15), 1-11.

Boj, S. F., Hwang, C., Baker, L. A., In, I., Chio, C., Engle, D. D., ... Tuveson, D. A. (2015). Resource Organoid Models of Human and Mouse Ductal Pancreatic Cancer. Cell, 160 324-338.

Castrogiovanni, C., Waterschoot, B., De Backer, O., \& Dumont, P. (2018). Serine 392 phosphorylation modulates p53 mitochondrial translocation and transcription independent apoptosis. Cell Death and Differentiation, 25(1), 1-14.

Chen, L., Manautou, J. E., \& Rasmussen, T. P. (2019). Development of precision medicine approaches based on inter-individual variability of BCRP /ABCG2. Acta Pharmaceutica Sinica $B, 9(4)$, 659-674.

Conroy, T., Desseigne, F., Ychou, M., Bouché, O., Guimbaud, R., Bécouarn, Y.,...Ducreux, M. (2011). FOLFIRINOX versus Gemcitabine for Metastatic Pancreatic Cancer. The New England Journal of Medecine, 364;19. 
Conroy, T., Hammel, P., Hebbar, M., Abdelghani, M. Ben, Wei, A. C., Raoul, J., ... Juzyna, B. (2018). FOLFIRINOX or Gemcitabine as Adjuvant Therapy for Pancreatic Cancer. The New England Journal of Medecine, 379;25.

De Man, FM., Goey AKL, Schaik RHN Van. (2018) Individualization of Irinotecan Treatment: A Review of Pharmacokinetics, Pharmacodynamics, and Pharmacogenetics. Clin Pharmacokinet 57(10):1229-54.

Descarpentries, C., Leprêtre, F., Sebda, S., Baldacci, S., \& Grégoire, V. (2018). Optimization of Routine Testing for MET Exon 14 Splice Site Mutations in NSCLC Patients. Journal of Thoracic Oncology, 13(12), 1873-1883.

Deyme, L., Barbolosi, D., Gattacceca, F. (2019). Population pharmacokinetics of FOLFIRINOX: a review of studies and parameters. Cancer Chemother Pharmacol.83. 27-42.

El Amrani, M., Fulbert, M., Gnemmi, V., Truant, S., Skrzypczyk, C., Tulasne, D., ... Huet, G. (2019). Gemcitabine - induced epithelial - mesenchymal transition - like changes sustain chemoresistance of pancreatic cancer cells of mesenchymal - like phenotype. Molecular Carcinogenesis (July), 1985-1997.

Ercan, G., Karlitepe A., Ozpolat, B. (2017). Pancreatic Cancer Stem Cells and Therapeutic Approaches. Anticancer Research 37: 2761-2775.

Erenpreisa, J., \& Cragg, M. S. (2013). Three steps to the immortality of cancer cells: senescence, polyploidy and self-renewal. Cancer Cell International, 13(1), 1.

Faubert, B., Boily, G., Izreig, S., Griss, T., Samborska, B., Dong, Z., ... Jones, R. G. (2013). Article AMPK Is a Negative Regulator of the Warburg Effect and Suppresses Tumor Growth In Vivo. CMET, 17(1), 113-124.

Fujii, M., \& Sato, T. (n.d.). Somatic cell-derived organoids as prototypes of human epithelial tissues and diseases. Nature Materials.

Guo, X., Goessl, E., Jin, G., Collie-duguid, E. S. R., Cassidy, J., Wang, W., \& Brien, V. O. (2008). Cell Cycle Perturbation and Acquired 5-Fluorouracil Chemoresistance, 14, 9-14.

Harada, H., Andersen, J. S., Mann, M., Terada, N., \& Korsmeyer, S. J. (2001). p70S6 kinase signals cell survival as well as growth, inactivating the pro-apoptotic molecule BAD. Proceedings of the National Academy of Sciences of the United States of America, 98(17), 9666-9670.

Hof, L., Moreth, T., Koch, M., Liebisch, T., Kurtz, M., Tarnick, J., ... Pampaloni, F. (2021). Long-term live imaging and multiscale analysis identify heterogeneity and core principles of epithelial organoid morphogenesis. BMC Biology, 1-22.

Huch, M., Bonfanti, P., Boj, S. F., Sato, T., Loomans, C. J. M., Wetering, M. Van De, ... Clevers, H. (2013). Unlimited in vitro expansion of adult bi-potent pancreas progenitors through the Lgr5 / R-spondin axis. The EMBO Journal, 32(20), 2708-2721.

Kaufmann, S. H., Desnoyers, S., Ottaviano, Y., Davidson, N. E., \& Poirier, G. G. (1993). Specific Proteolytic Cleavage of Poly(ADP-ribose) Polymerase: An Early Marker of Chemotherapy-induced Apoptosis. Cancer Research, 53(17), 3976-3985. 
Lee, S. H., Kim, P. H., \& Hwang, J. (2014). CD24 and S100A4 Expression in Resectable Pancreatic Cancers With Earlier Disease Recurrence and Poor Survival. Pancreas Journal 43(3), 380-388.

Leushacke, M., \& Barker, N. (2012). Lgr5 and Lgr6 as markers to study adult stem cell roles in self-renewal and cancer. Oncogene, 3009-3022.

Loomans, C., Giuliani N.W., Balak J., Ringnalda F., Van Gurp L., Huch M., Boj S.F., Sato T., et al. (2018). Expansion of Adult Human Pancreatic Tissue Yields Organoids Harboring Progenitor Cells with Endocrine Differentiation Potential. Stem Cell Reports.712-724.

Mahon, P. C., Baril, P., Bhakta, V., Chelala, C., Caulee, K., Harada, T., \& Lemoine, N. R. (2007). S100A4 Contributes to the Suppression of BNIP3 Expression, Chemoresistance , and Inhibition of Apoptosis in Pancreatic Cancer. Cancer Res (14), 6786-6796.

Mathijssen, R.H.J., Robbert J. van Alphen, Verweij J., Loos W.J., Nooter K., Stoter G., and Sparreboom A. (2001) Clinical pharmacokinetics and metabolism of nimesulide. Inflammopharmacology. 9(1-2):81-9.

Mashimo, M., Onishi, M., Uno, A., Tanimichi, A., Nobeyama, A., Mori, M., ... Fujii, T. (2020). The 89-kDa PARP1 cleavage fragment serves as a cytoplasmic PAR carrier to induce AIF-mediated apoptosis. Journal of Biological Chemistry, jbc.RA120.014479.

Mizrahi, J. D., Surana, R., Valle, J. W., \& Shroff, R. T. (2020). Pancreatic cancer. The Lancet, 395(10242), 2008-2020.

Moon, H. H., Kim, S. H., \& Ku, J. L. (2016). Correlation between the promoter methylation status of ATP-binding cassette sub-family $\mathrm{G}$ member 2 and drug sensitivity in colorectal cancer cell lines. Oncology Reports, 35(1), 298-306.

Muriithi, W., Macharia, L. W., Heming, C. P., Echevarria, J. L., Filho, P. N., \& Neto, V. M. (2020). REVIEW ABC transporters and the hallmarks of cancer: roles in cancer aggressiveness beyond multidrug resistance $A B C$ proteins: structure and function dynamics. Cancer Biol Med, 17: 253-269.

Murphy, J.E., Wo, J.Y. and Ryan, D.P.(2018).Total Neoadujuvant therapy with FOLFIRINOX followed by individualized chemoradiotherapy for bordeline resectable pancreatic adenocarcinoma. JAMA Oncol. 963-969

Nagle, P. W., Plukker, J. T. M., Muijs, C. T., van Luijk, P., \& Coppes, R. P. (2018). Patientderived tumor organoids for prediction of cancer treatment response. Seminars in Cancer Biology, 53(May), 258-264.

Nastase, A., Dima, S., Tica, V., Florea, R., Sorop, A., llie, V., ... Diaconu, C. C. (2016). S100A4 and ERBB2 as Co-Factors in Pancreatic Cancer. Journal of Translational Medicine and Research, 21(4), 259-266.

Pasch, C. A., Favreau, P. F., Yueh, A. E., Babiarz, C. P., Gillette, A. A., Sharick, J. T., ... Deming, D. A. (2019). Patient-derived cancer organoid cultures to predict sensitivity to chemotherapy and radiation. Clinical Cancer Research, 25(17), 5376-5387.

Podhorecka, M., Skladanowski, A., \& Bozko, P. (2010). H2AX phosphorylation: Its role in DNA damage response and cancer therapy. Journal of Nucleic Acids, 2010. 
Praharaj, P. P., Bhutia, S. K., Nagrath, S., Bitting, R. L., \& Deep, G. (2018). Circulating tumor cell-derived organoids: Current challenges and promises in medical research and precision medicine. Biochimica et Biophysica Acta - Reviews on Cancer, 1869(2), 117127.

Rahib, L., Smith, B. D., Aizenberg, R., Rosenzweig, A. B., Fleshman, J. M., \& Matrisian, L. M. (2014). Projecting Cancer Incidence and Deaths to 2030 : The Unexpected Burden of Thyroid, Liver, and Pancreas Cancers in the United States. Cancer Res, 74:29132921.

Romero-Calvo, I., Weber, C. R., Ray, M., Brown, M., Kirby, K., Nandi, R. K., ... White, K. P. (2019). Human organoids share structural and genetic features with primary pancreatic adenocarcinoma tumors. Molecular Cancer Research, 17(1), 70-83.

Rosner, G. L., Panetta, J. C., Innocenti, F., \& Ratain, M. J. (2008). Pharmacogenetic Pathway Analysis of Irinotecan, CliniCal pharmaCology \& TherapeuTiCs 84(3), 393402.

Rosty, C., Ueki, T., Argani, P., Jansen, M., Yeo, C. J., Cameron, J. L., ... Goggins, and M. (2002). Overexpression of S100A4 in Pancreatic Ductal Adenocarcinomas Is Associated with Poor Differentiation and DNA Hypomethylation. AJP 160(1), 45-50.

Sacchi, G. (1971). Upper and lower bounds for the optimum design of structures with random resistance. Meccanica, 6(3), 168-171.

Sasaki, N., Ishiwata, T., Hasegawa, F., Michishita, M., Kawai, H., Matsuda, Y., ... Toyoda, M. (2018). Stemness and anti-cancer drug resistance in ATP-binding cassette subfamily G member 2 highly expressed pancreatic cancer is induced in 3D culture conditions. Cancer Science, 109(4), 1135-1146.

Shah, S. A., Potter, M. W., Ricciardi, R., Perugini, R. A., \& Callery, M. P. (2001). FRAPp70s6K Signaling Is Required for Pancreatic Cancer Cell Proliferation. J Surg Res 130, $123-130$.

Sharom, F. J. (2008) ABC multidrug transporters: Structure, function and role in chemoresistance. Pharmacogenomics 9(1), 105-127.

Shin, W., Wu, A., Min, S., Fleming, D., Eckhardt, G., Shin, W., ... Eckhardt, S. G. (2020). iScience II Spatiotemporal Gradient and Instability of Wnt Induce Heterogeneous Growth and Differentiation of Human Intestinal Organoids Induce Heterogeneous Growth and Differentiation of Human Intestinal Organoids. ISCIENCE, 23(8), 101372.

Shukla, S. K., Purohit, V., Mehla, K., Cantley, L. C., Berim, L., \& Singh, P. K. (2017). Anabolic Glucose Metabolism to Impart Gemcitabine Resistance to Pancreatic Cancer Article MUC1 and HIF-1alpha Signaling Crosstalk Induces Anabolic Glucose Metabolism to Impart Gemcitabine Resistance to Pancreatic Cancer. Cancer Cell, 7187.

Skrypek, N. (2013). The MUC4 mucin mediates gemcitabine resistance of human pancreatic cancer cells via the Concentrative Nucleoside Transporter family. Oncogene, 17141723.

Skrypek, N., Vasseur, R., Vincent, A., Duchêne, B., Seuningen, I. Van, \& Jonckheere, N. (2015). The oncogenic receptor ErbB2 modulates gemcitabine and irinotecan / SN-38 
chemoresistance of human pancreatic cancer cells via hCNT1 transporter and multidrug-resistance associated protein MRP-2. Oncotarget 6(13), 10853-10867.

Soledad, M., Varela, R., Mucci, S., Agustín, G., Richardson, V., Hanon, O. M., ... Romorini, L. (2018). Regulation of cyclin E1 expression in human pluripotent stem cells and derived neural progeny. Cell Cycle, 17(14), 1719-1742.

Sun, Q., Ye, Z., Qin, Y., Fan, G., Ji, S., Zhuo, Q., .. Yu, X. (2020). Oncogenic function of TRIM2 in pancreatic cancer by activating ROS-related NRF2 / ITGB7 / FAK axis. Oncogene, 6572-6588.

Tadros, S., Shukla, S. K., King, R. J., Gunda, V., Vernucci, E., Abrego, J., ... Singh, P. K. (2017). De Novo Lipid Synthesis Facilitates Gemcitabine Resistance through Endoplasmic Reticulum Stress in Pancreatic Cancer. Cancer Research, 5503-5518.

Taira, N., Nihira, K., Yamaguchi, T., Miki, Y., \& Yoshida, K. (2007). DYRK2 Is Targeted to the Nucleus and Controls p53 via Ser46 Phosphorylation in the Apoptotic Response to DNA Damage. Molecular Cell, 25(5), 725-738.

Toledo, F., Wahl, G.M.(2006) Regulating the p53 pathway : in vitro hypotheses, in vivo veritas. Nature Reviews Cancer Vol 6 issue 12, p909-15.

Toyoda, Y., Takada, T., \& Suzuki, H. (2019). Inhibitors of human ABCG2: From technical background to recent updates with clinical implications. Frontiers in Pharmacology, 10(MAR), 1-9.

Tsuboi, M., Taniuchi, K., Furihata, M., Naganuma, S., Kimura, M., Watanabe, R., ... Saibara, T. (2016). Pancreatology Vav3 is linked to poor prognosis of pancreatic cancers and promotes the motility and invasiveness of pancreatic cancer cells. Pancreatology, 16(5), 905-916.

Valenzuela, B., Nalda-Molina, R., Bretcha-Boix, P., Escudero-Ortíz, V., Duart, M. J., Carbonell, V., ... Pérez-Ruixo, J. J. (2011). Pharmacokinetic and pharmacodynamic analysis of hyperthermic intraperitoneal oxaliplatin-induced neutropenia in subjects with peritoneal carcinomatosis. AAPS Journal, 13(1), 72-82.

Vincent, A., Ducourouble, M., \& Van Seuningen, I. (2008). Epigenetic regulation of the human mucin gene MUC4 in epithelial cancer cell lines involves both DNA methylation and histone modifications mediated by DNA methyltransferases and histone deacetylases. The FASEB Journal, 22(8), 3035-3045.

Vincent, A., Herman, J., Schulick, R., Hruban, R. H., \& Goggins, and M. (2011). Pancreatic cancer: An overview. LANCET, 11(2), 168-180.

Wu, D., \& Pan, W. (2009). GSK3: a multifaceted kinase in Wnt signaling. Trends in Biochemical Sciences, 35(3), 161-168. https://doi.org/10.1016/j.tibs.2009.10.002

Xu, G., Kwon, G., Cruz, W. S., Marshall, C. A., \& Mcdaniel, M. L. (n.d.). (2001). Metabolic Regulation by Leucine of Translation Initiation Through the mTOR-Signaling Pathway by Pancreatic Cells. Diabetes (25), 353-360. 
Yang, C., Xia, B. R., Jin, W. L., \& Lou, G. (2019). Circulating tumor cells in precision oncology: Clinical applications in liquid biopsy and 3D organoid model. Cancer Cell International, 19(1), 1-13.

Zhang, J., Deng, X., \& Kahle, K. T. (2016). Leveraging unique structural characteristics of WNK kinases to achieve therapeutic inhibition. Science Signaling, 9(450), 1-4.

Zhou, C., Sun, H., Zheng, C., Gao, J., Fu, Q., Hu, N.,... Lyu, J. (2018). Oncogenic HSP60 regulates mitochondrial oxidative phosphorylation to support Erk1/2 activation during pancreatic cancer cell growth. Cell Death and Disease, 9(2), 161. 


\section{Graphical Abstract}

Patient-Derived tumour organoids (PDO) have emerged as a critical and useful approach to preserve tumour cell heterogeneity in primary culture. This work introduces the first genuine PDO model of acquired resistance to the three drugs combined in the FOLFIRINOX regimen (5-fluorouracil, irinotecan/SN-38 and oxalipatin), recapitulating as accurately as possible clinical aspects of pancreatic cancer chemoresistance. This model could pave the way for new therapeutic strategies.

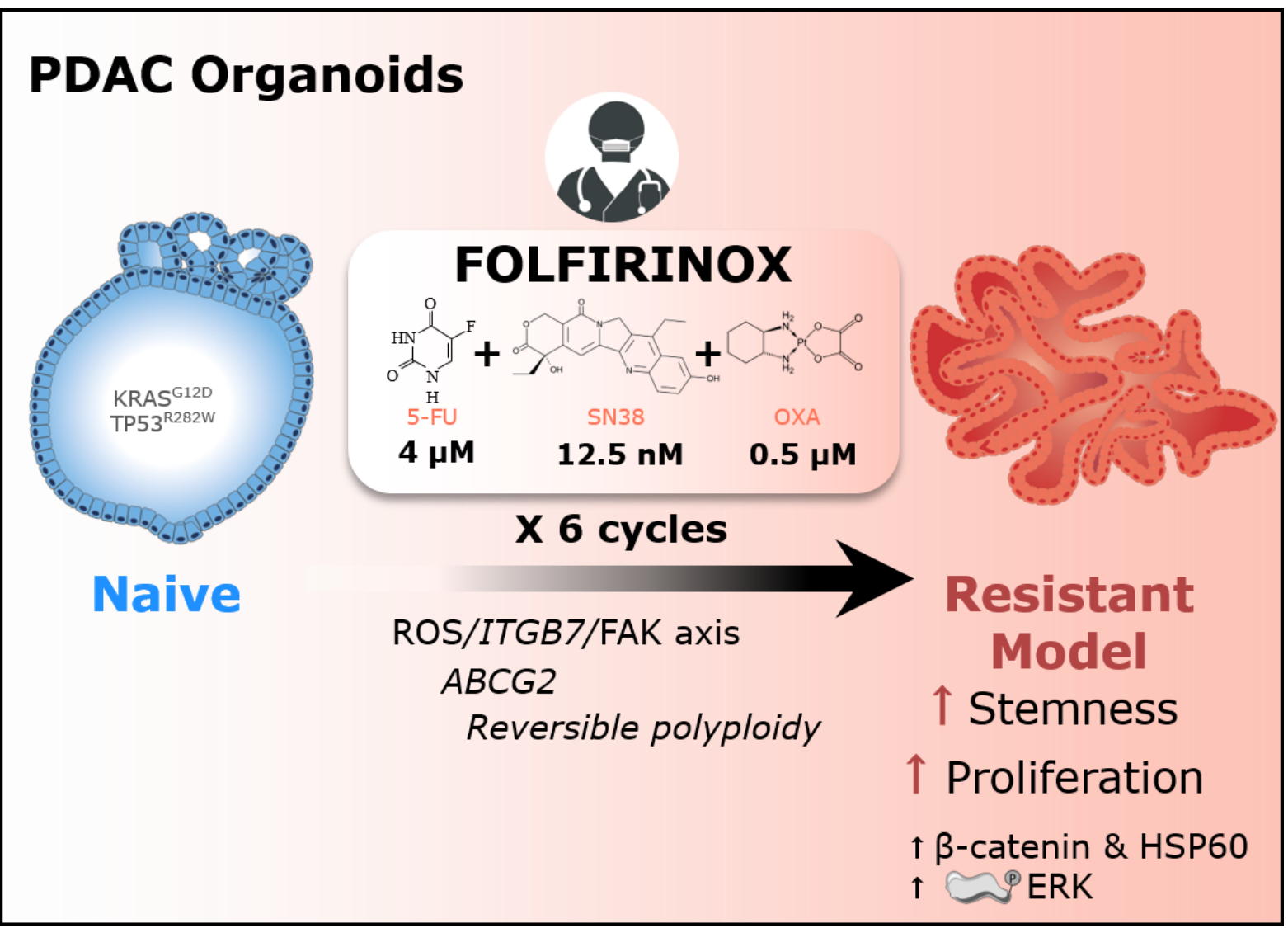




\section{Figure 1}
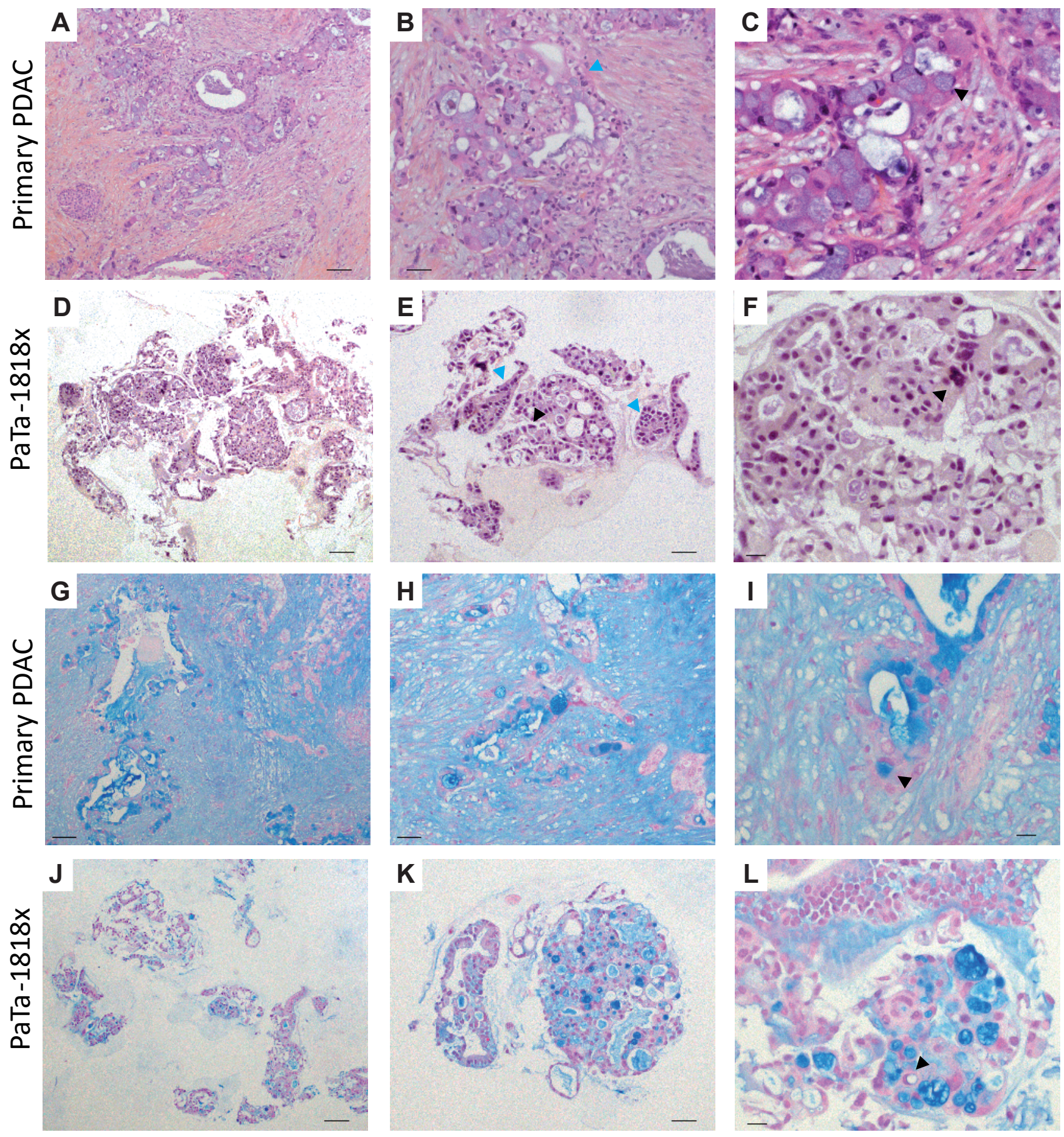

M

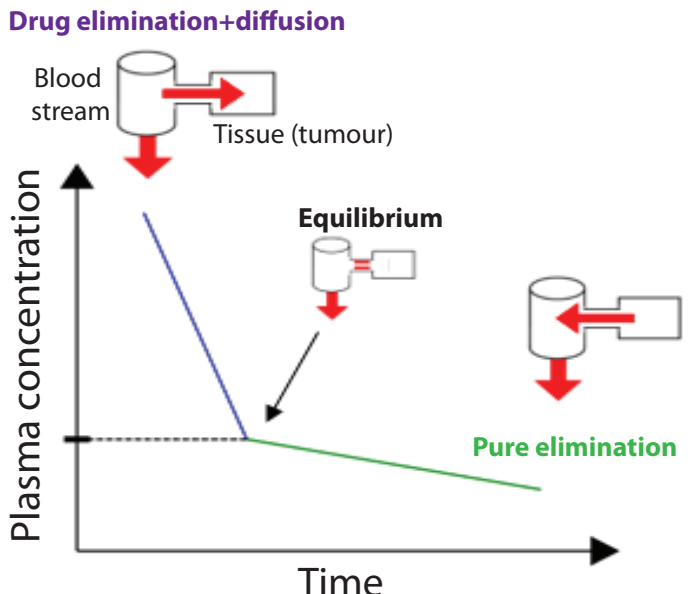

$\mathbf{N}$

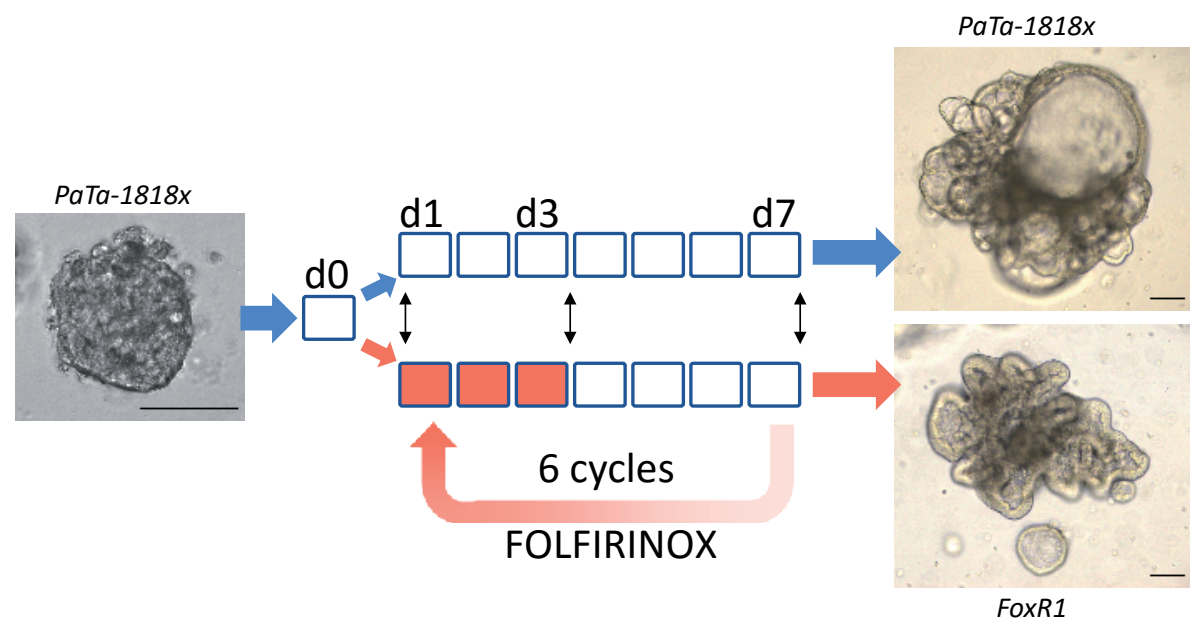


A
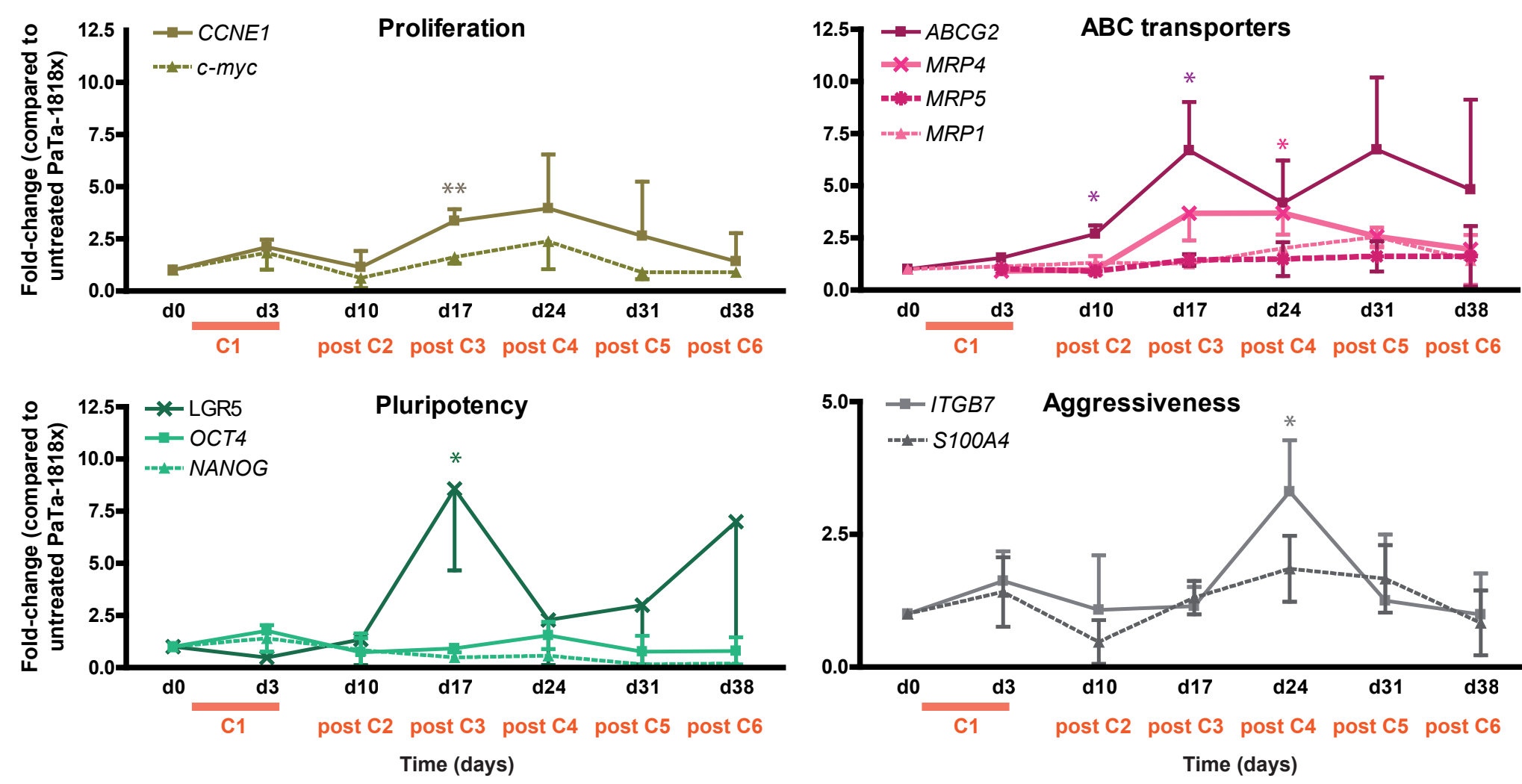

B
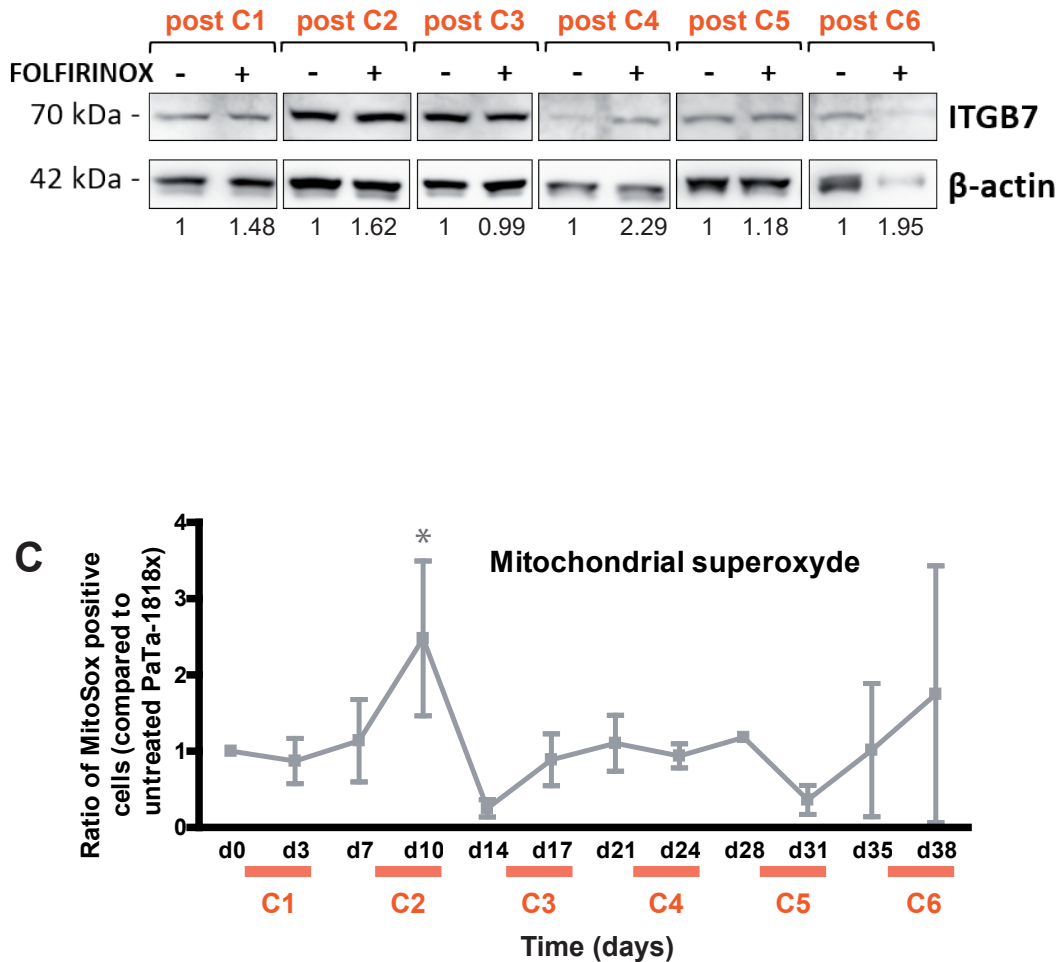
Figure 4

A

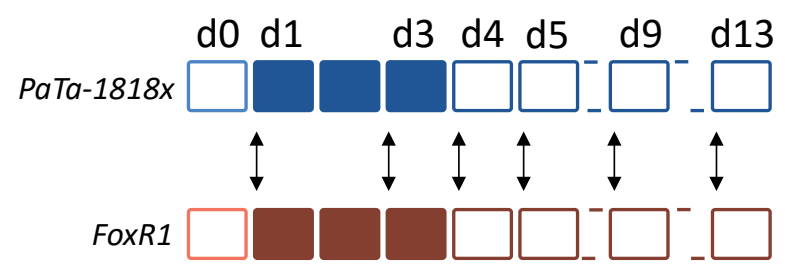

d1

d3

d4

d5

d9

d13

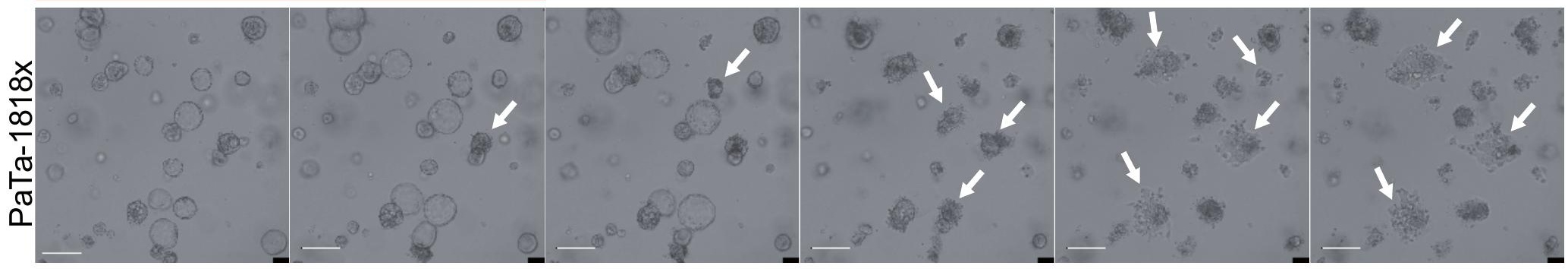

B

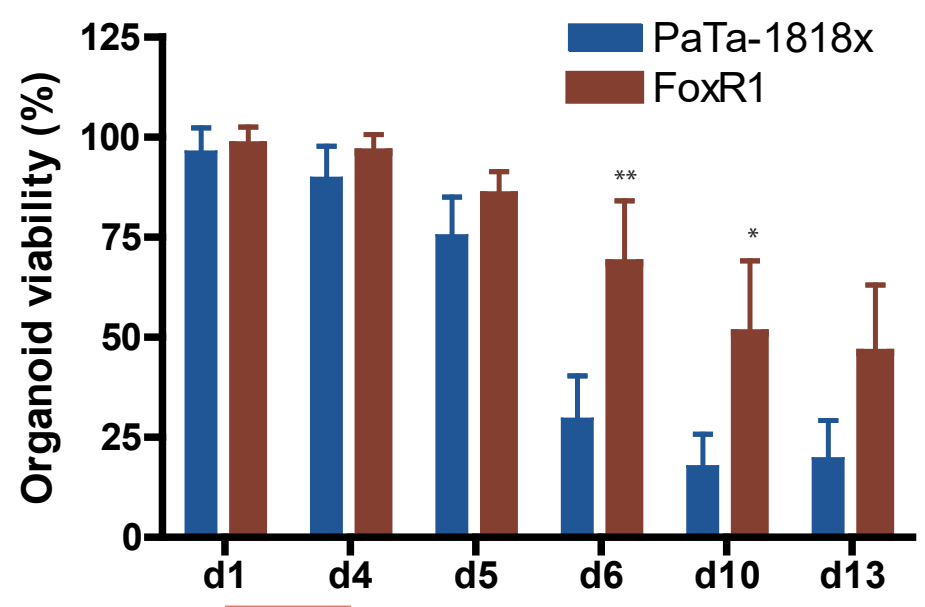

D
C

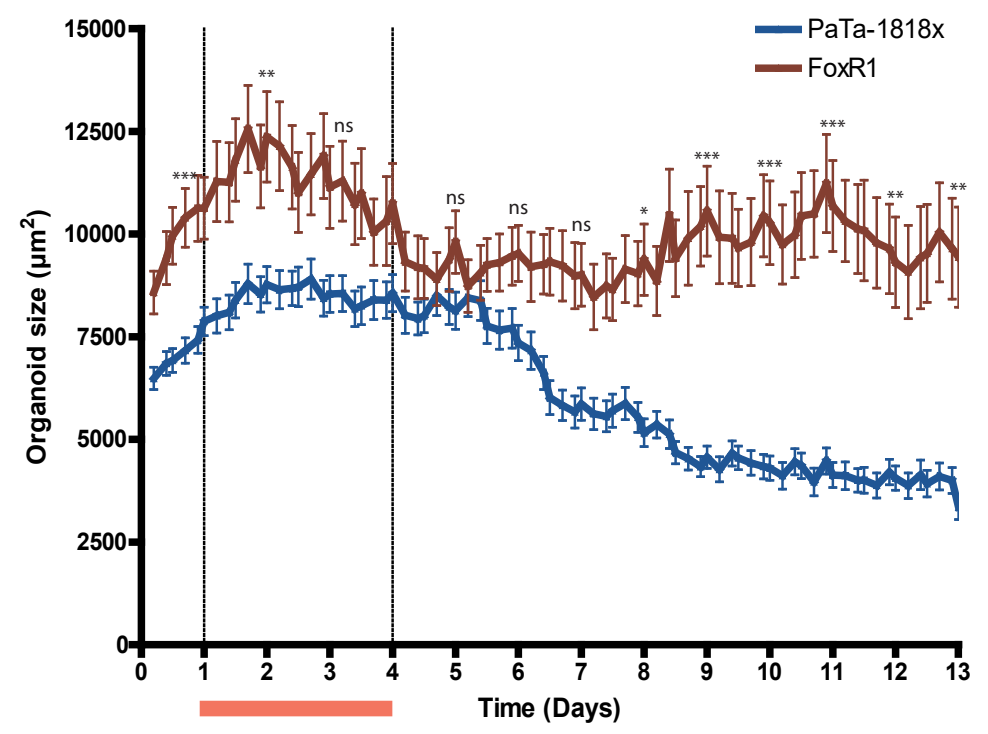

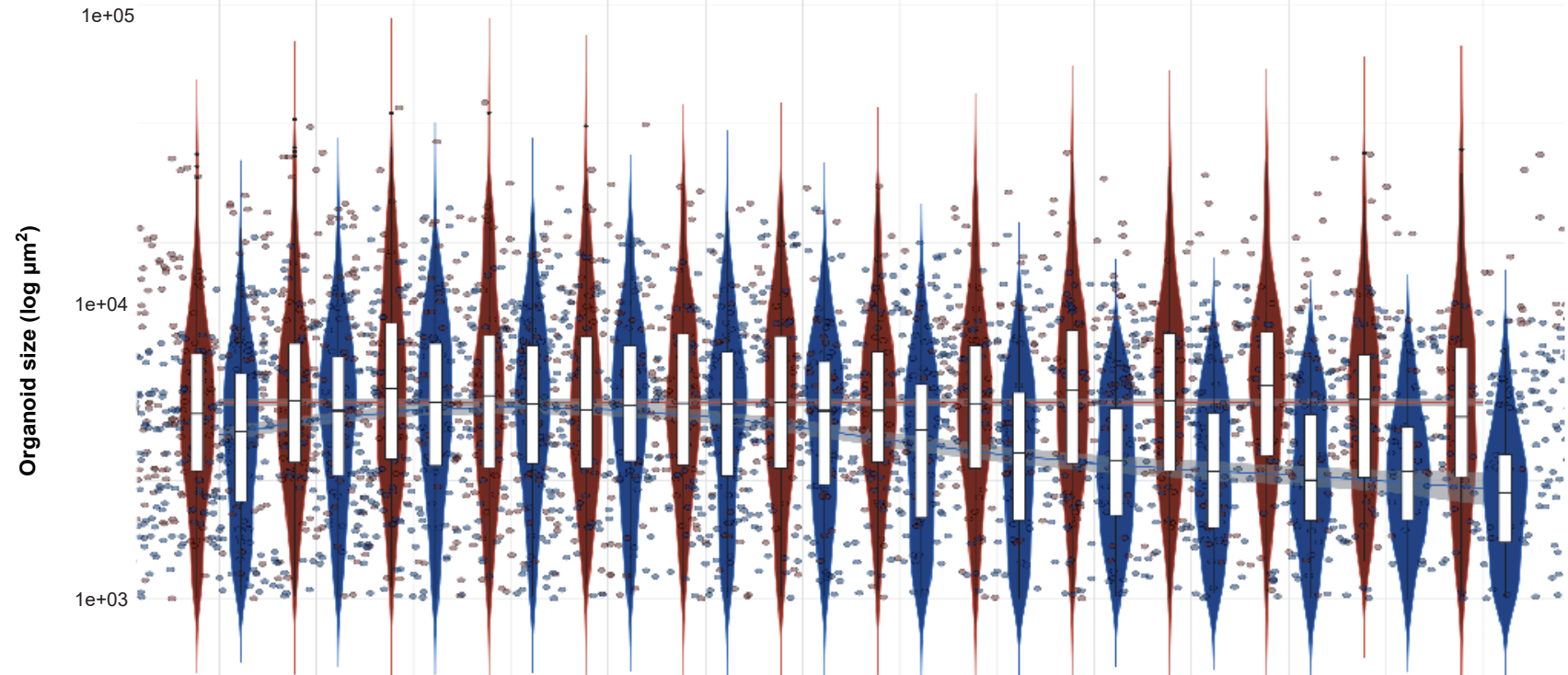

do 
Figure 5

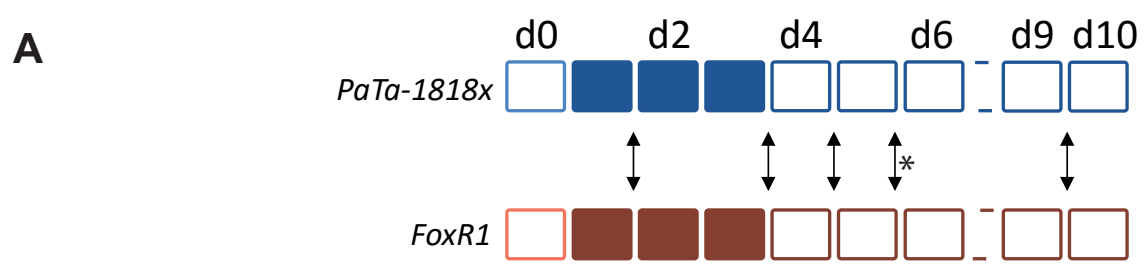

B

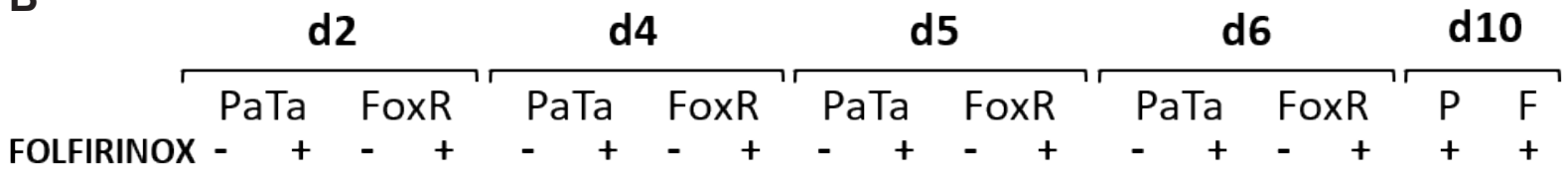

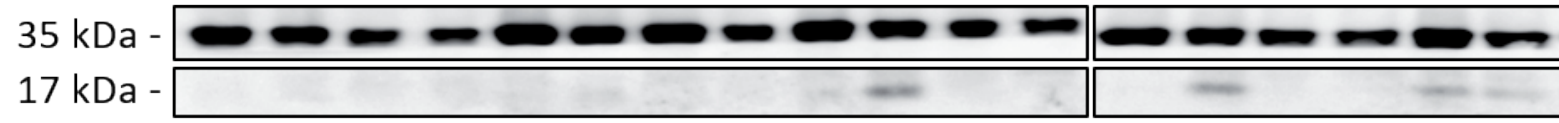

Caspase 3 (FL)

Cleaved Caspase 3

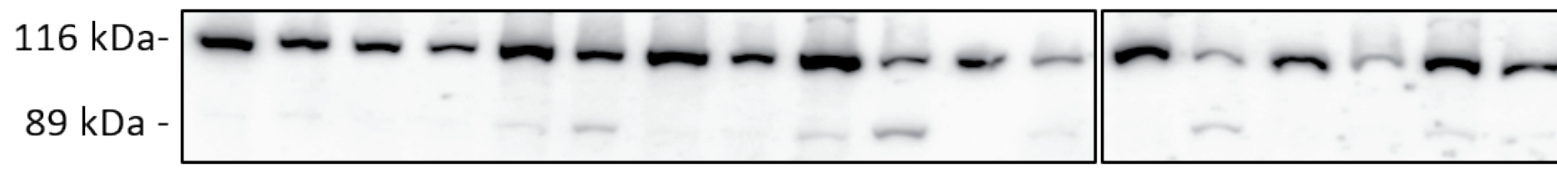

PARP (FL)

Cleaved PARP

$19 \mathrm{kDa}$

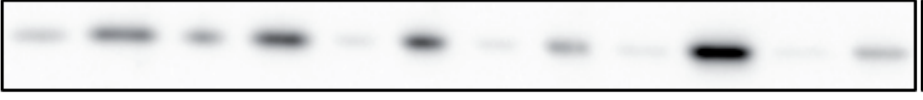

$--\infty-1$

PH2A.X

42 kDa -

$-\infty-\infty-\infty-a c t i n$
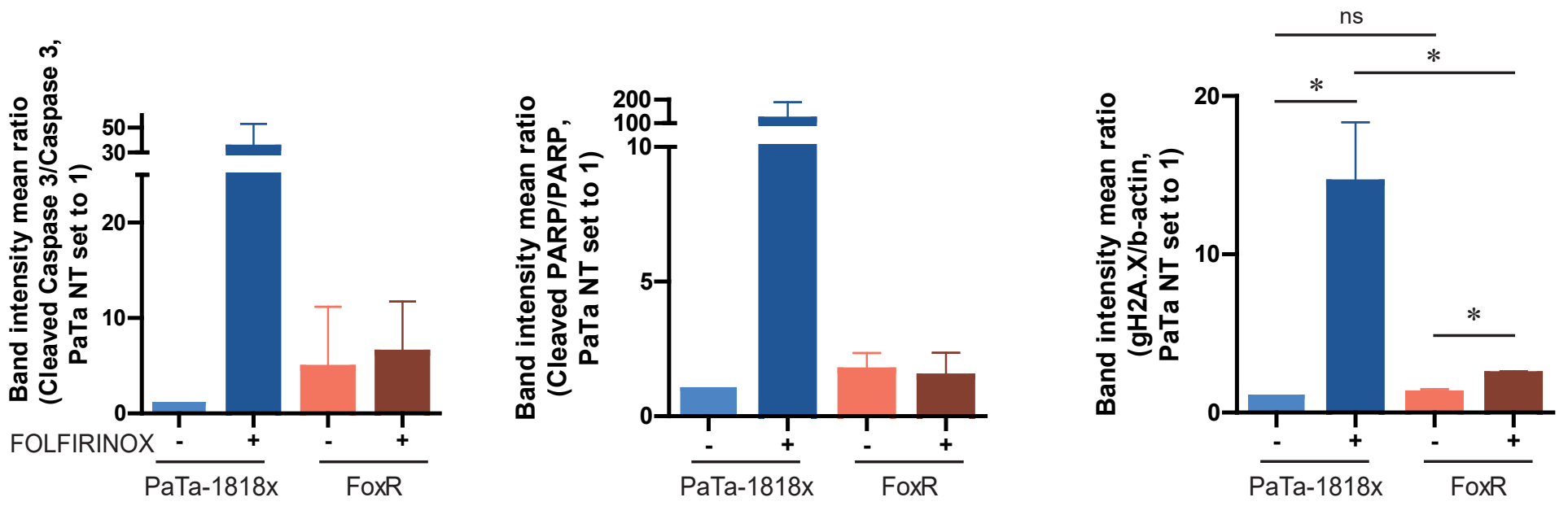

C

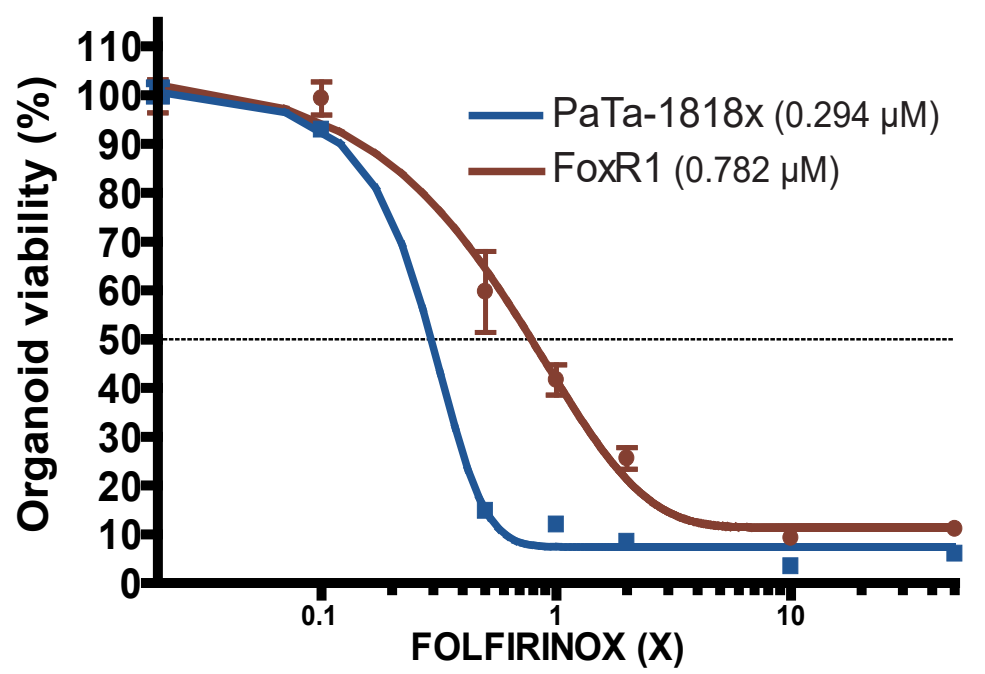


A

Figure 6

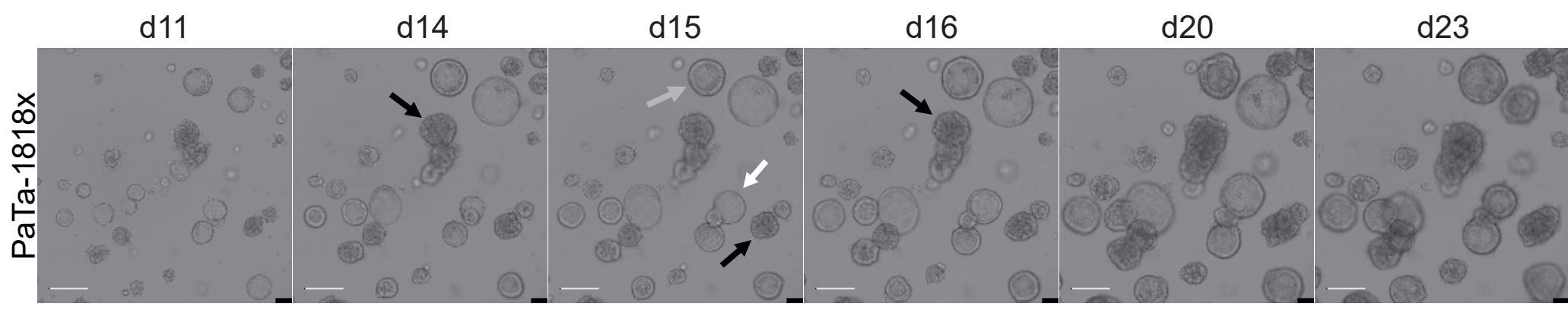

要

B

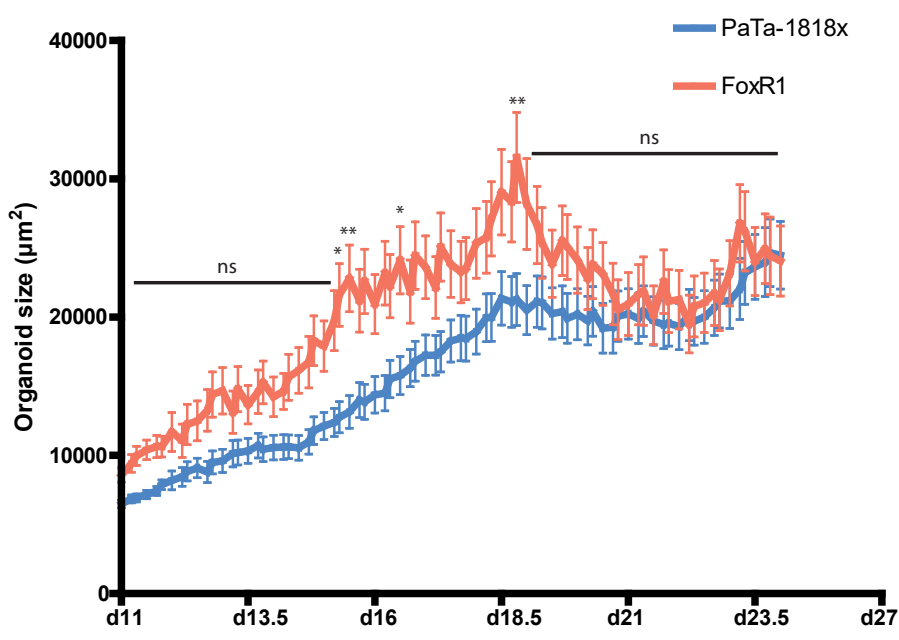

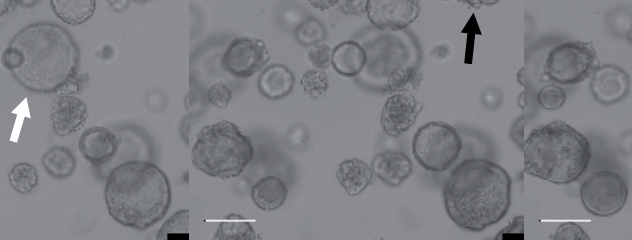

C

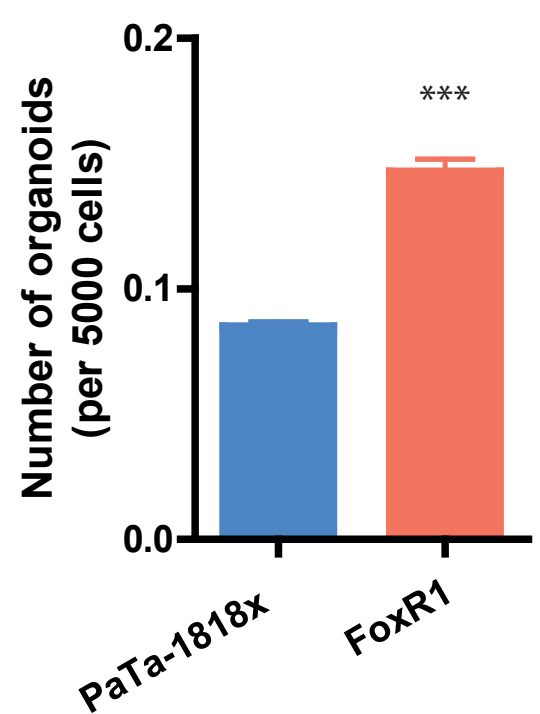

D

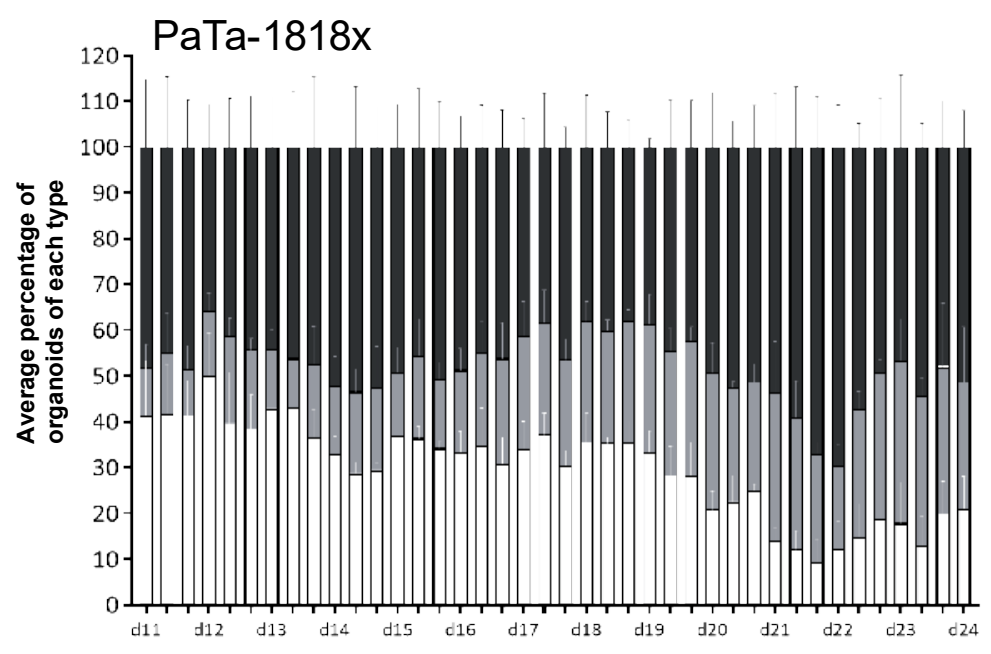

E

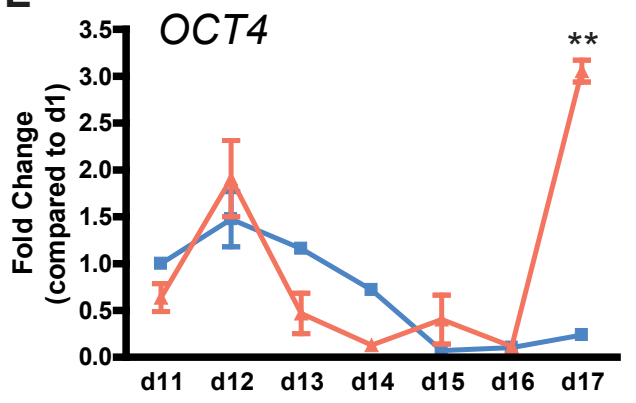

- Mature

$\square$ Intermediate

$\square$ Cystic
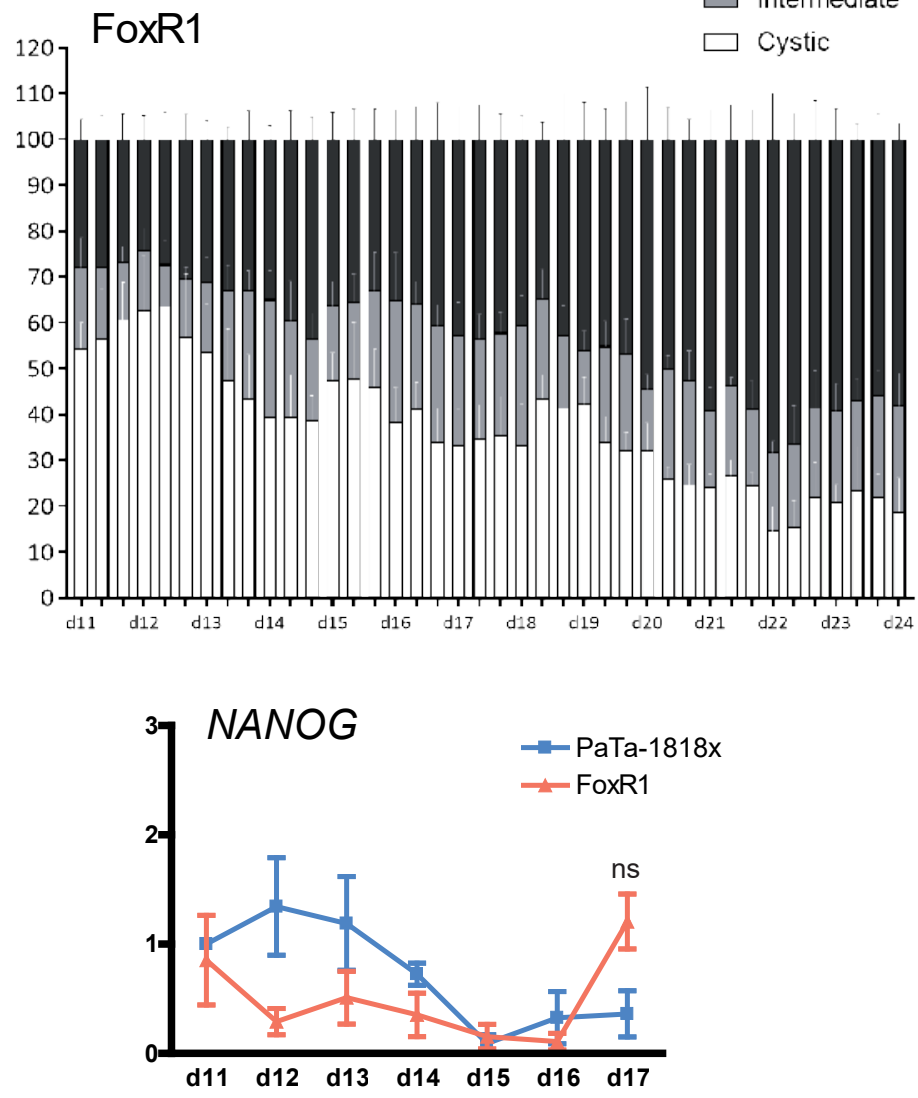
A

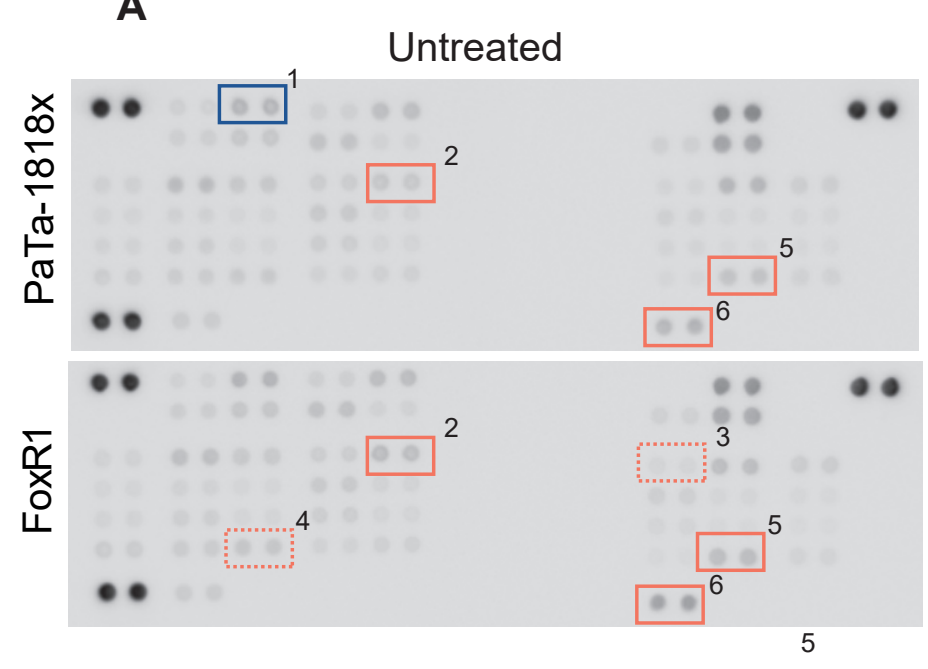

B

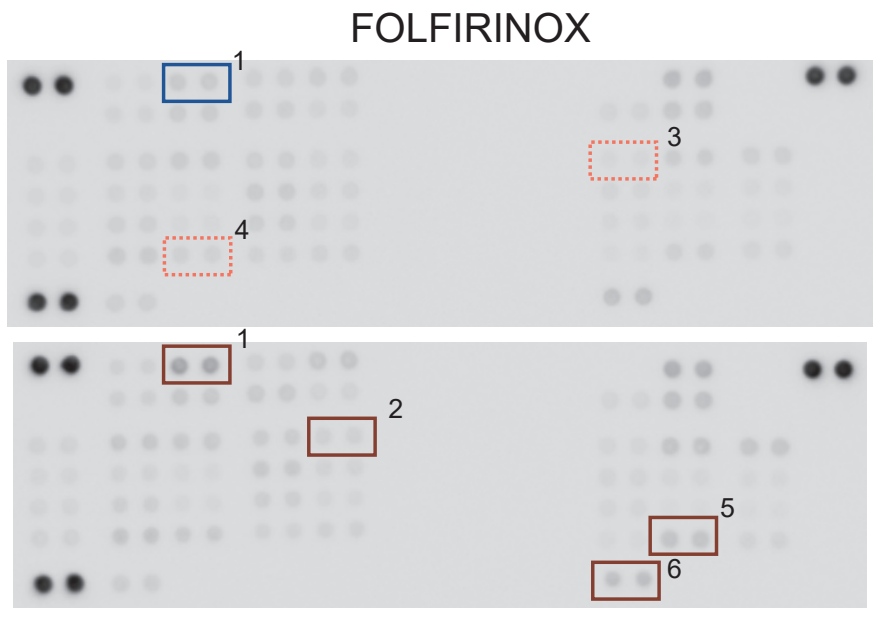

1: ERK1/2

2: $\beta$-catenin

3: p70 S6 kinase

4: FAK

5: WNK1

6: HSP60

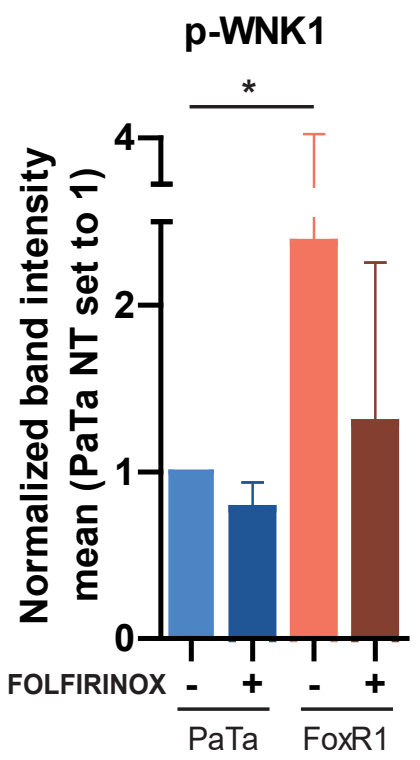

p-FAK

p-B-catenin

p-AKT

HSP60

p-ERK1/2

C

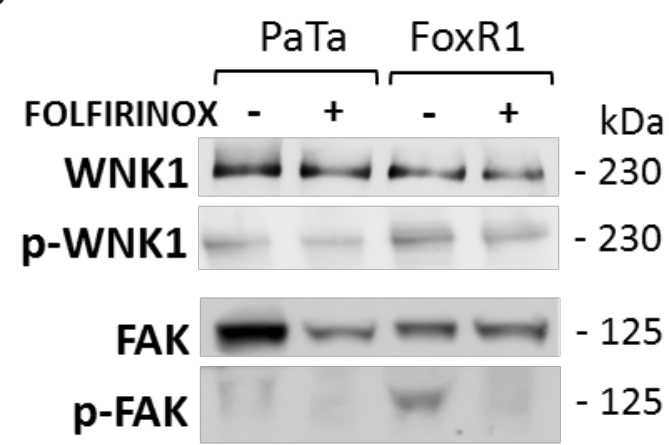

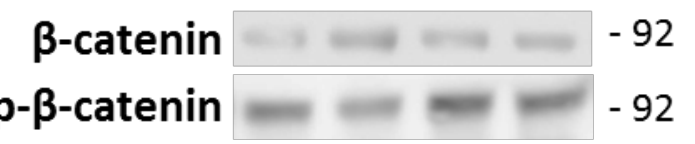

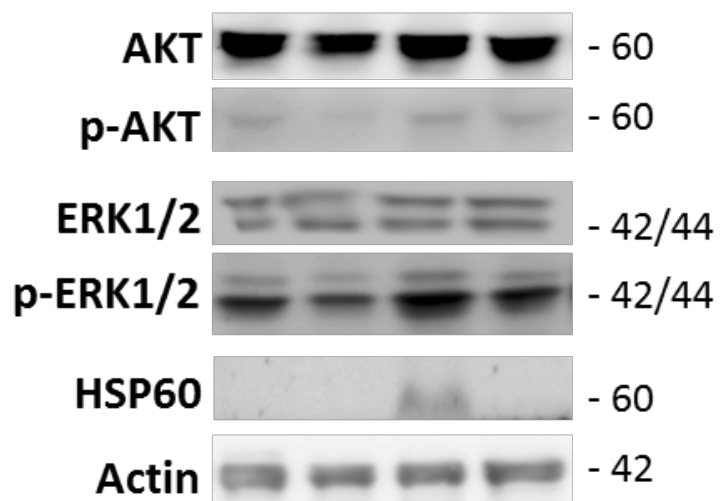

D

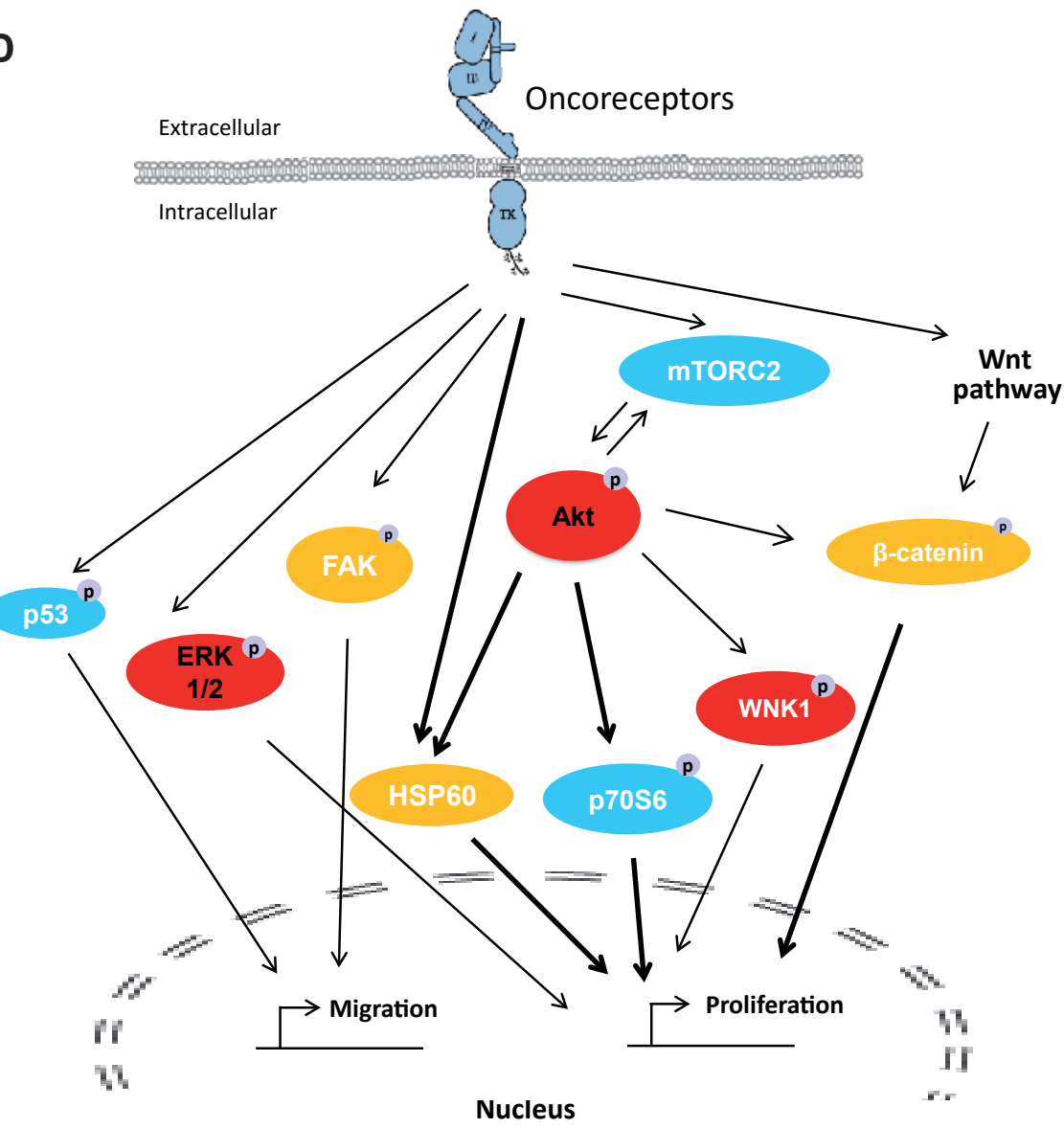


A

\section{Supplemental figure 1}
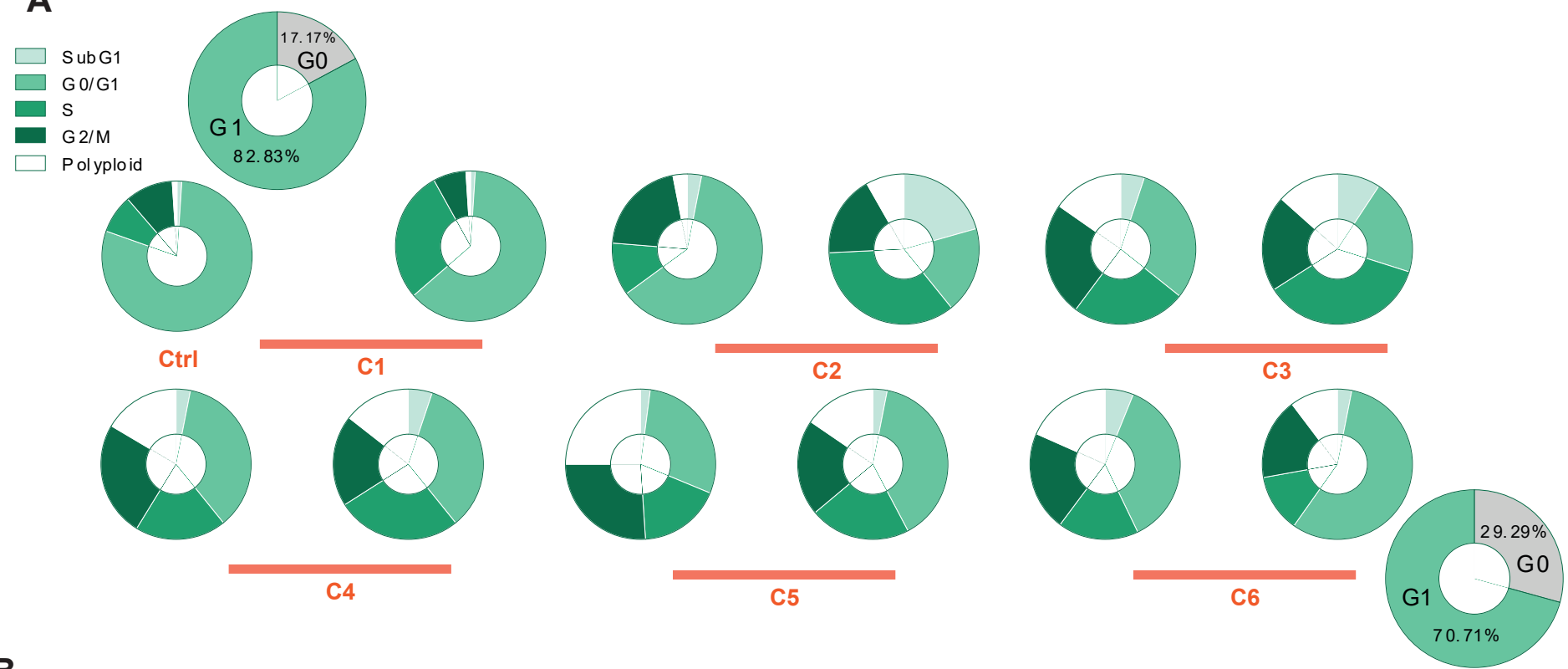

B

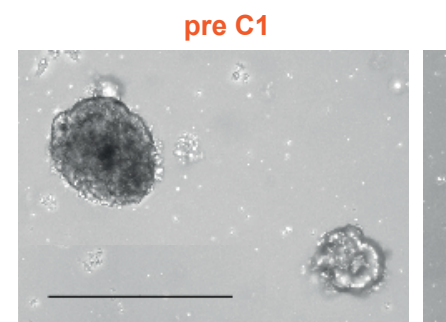

post C1

post $\mathrm{C} 2$

post C3
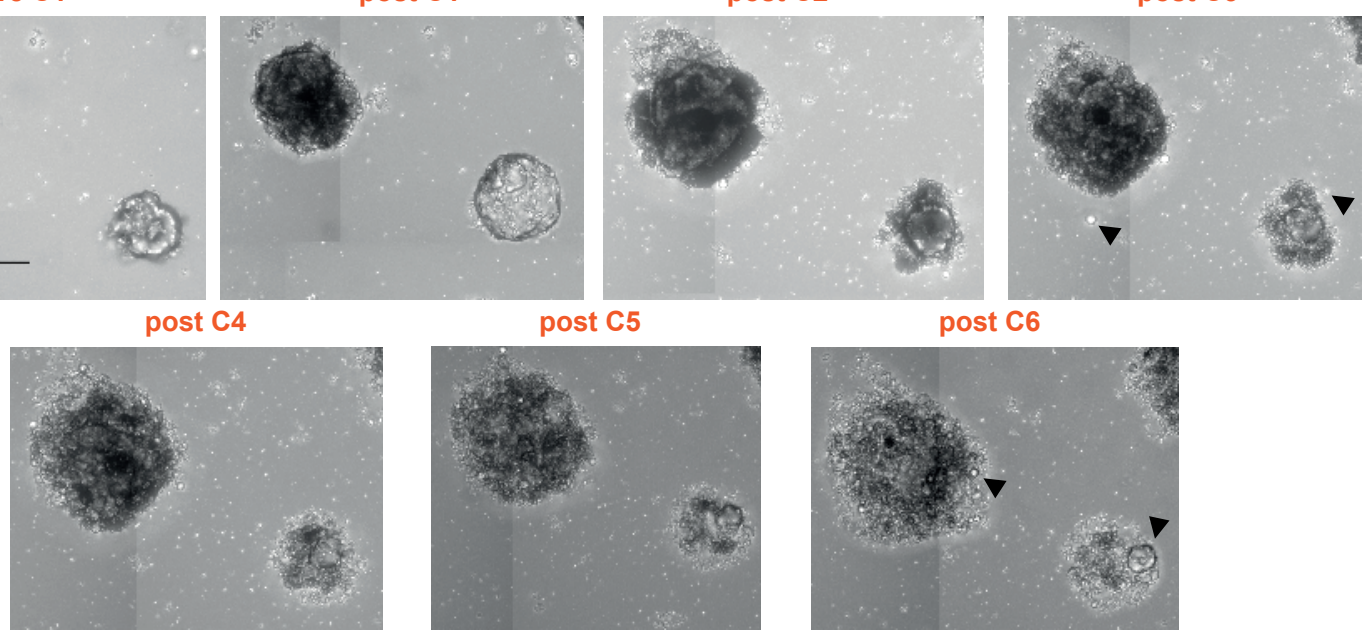

C

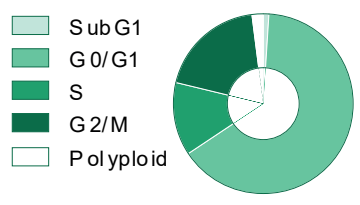

Ctrl

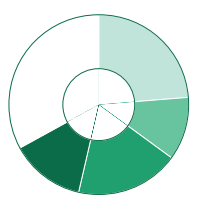

D
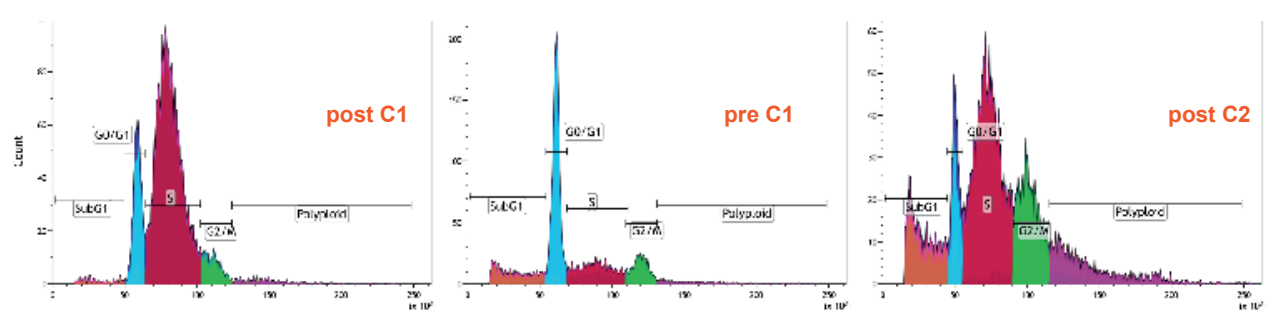
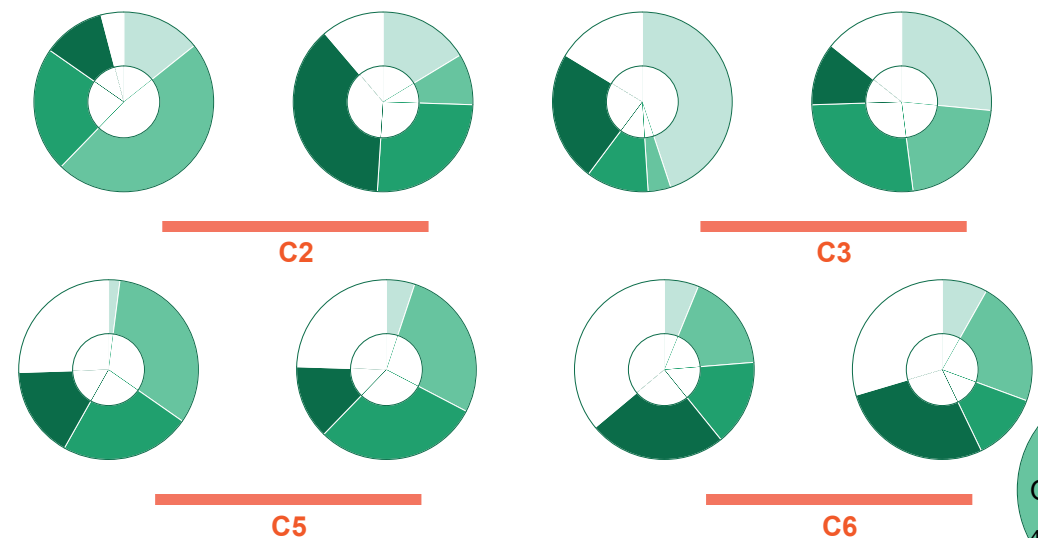

C6

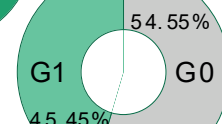

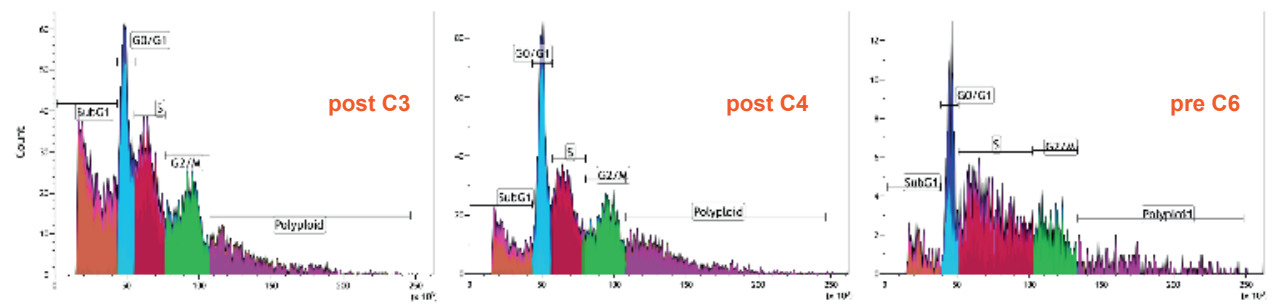




\section{Supplemental figure 3}

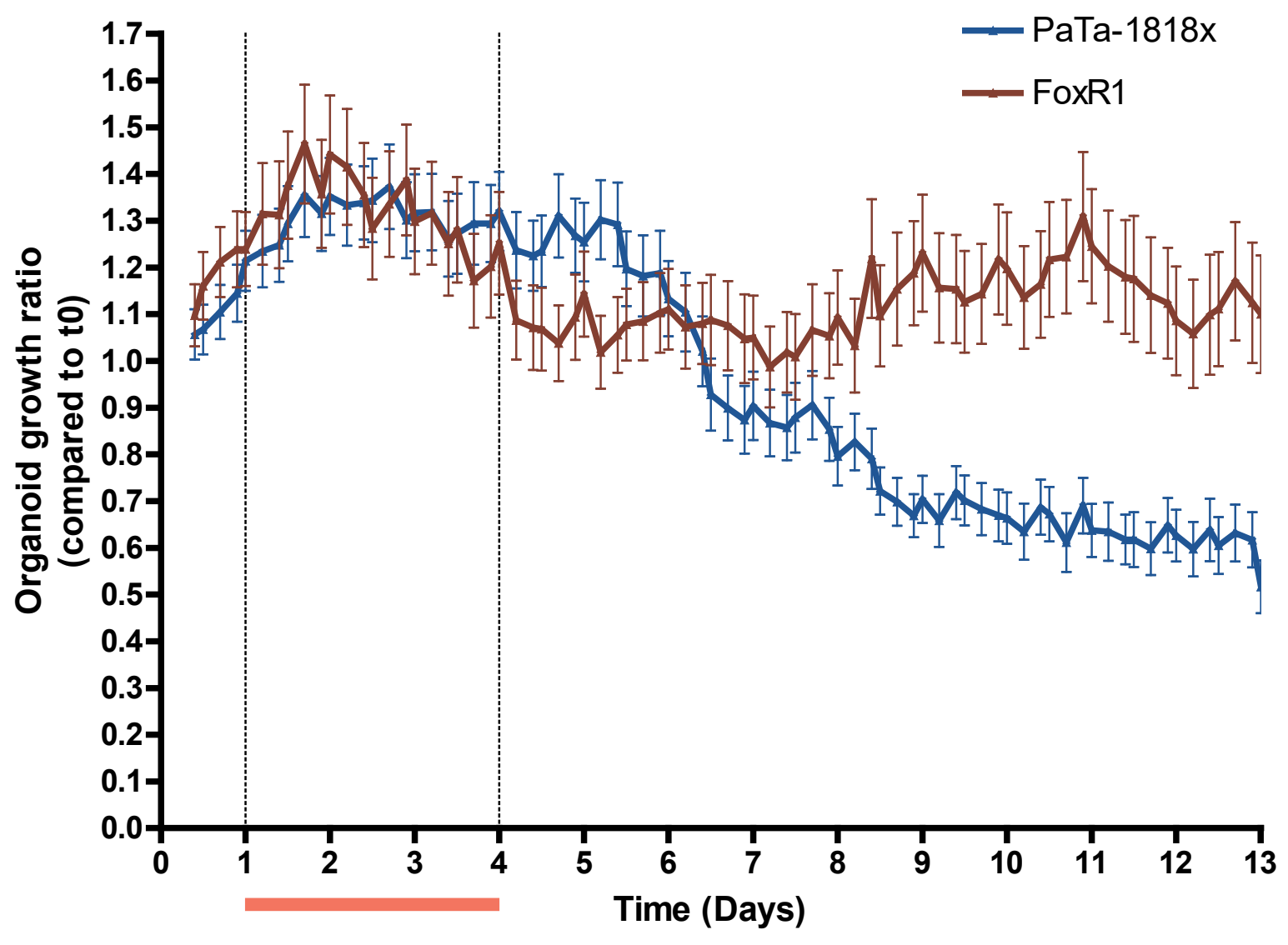


Supplemental table 1

\begin{tabular}{|c|c|c|c|c|c|}
\hline \multirow[b]{2}{*}{ Phosphoprotein } & \multicolumn{5}{|c|}{ Phosphorylation fold increase } \\
\hline & FoxR1 (NT)/PaTa (NT) & PaTa (T)/PaTa (NT) & FoxR1 (T)/FoxR1 (NT) & FoxR1 (T)/PaTa (T) & FoxR1 (NT)/PaTa (T) \\
\hline REF & 1,000 & 1,000 & 1,000 & 1,000 & 1,000 \\
\hline p38a & 1,069 & 0,919 & 0,933 & 1,086 & 1,164 \\
\hline ERK1/2 & 1,076 & 0,781 & 1,114 & 1,533 & 1,377 \\
\hline JNK1/2/3 & 1,101 & 1,000 & 0,929 & 1,023 & 1,101 \\
\hline GSK-3 $\alpha / \beta$ & 1,086 & 0,803 & 0,838 & 1,133 & 1,352 \\
\hline p53 (S392) & 0,988 & 0,589 & 0,680 & 1,139 & 1,676 \\
\hline REF & 1,039 & 0,971 & 1,027 & 1,099 & 1,070 \\
\hline EGFR & 1,117 & 0,936 & 0,891 & 1,063 & 1,193 \\
\hline MSK1/2 & 1,107 & 0,934 & 0,899 & 1,066 & 1,185 \\
\hline AMPKa1 & 1,118 & 0,852 & 0,837 & 1,098 & 1,313 \\
\hline Akt 1/2/3 (S473) & 1,103 & 0,993 & 0,935 & 1,039 & "1,111" \\
\hline Akt 1/2/3 (T308) & 1,089 & 0,938 & 0,881 & 1,023 & 1,161 \\
\hline p53 (S46) & 1,027 & 0,623 & 0,686 & 1,130 & 1,647 \\
\hline TOR & 1,082 & 0,939 & 0,924 & 1,065 & 1,152 \\
\hline CREB & 1,085 & 0,784 & 0,809 & 1,120 & 1,384 \\
\hline HSP27 & 1,099 & 0,966 & 0,904 & 1,028 & 1,138 \\
\hline AMPKa2 & 1,077 & 0,977 & 0,934 & 1,030 & 1,103 \\
\hline$\beta$-catenin & 1,246 & 0,818 & 0,703 & 1,070 & 1,523 \\
\hline p70S6 Kinase (T389) & 1,109 & 0,964 & 0,921 & 1,060 & 1,151 \\
\hline p53 (S15) & 1,007 & 0,749 & 0,865 & 1,163 & 1,345 \\
\hline c-Jun & 1,133 & 0,983 & 1,014 & 1,169 & 1,153 \\
\hline Src & 1,096 & 0,973 & 0,923 & 1,040 & 1,126 \\
\hline Lyn & 1,108 & 0,993 & 0,912 & 1,018 & 1,116 \\
\hline Lck & 1,103 & 0,974 & 0,919 & 1,041 & 1,132 \\
\hline STAT2 & 1,076 & 0,982 & 0,933 & 1,022 & 1,096 \\
\hline STAT5a & 1,094 & 0,993 & 0,930 & 1,024 & 1,101 \\
\hline p70S6 Kinase (T421/S424) & 1,139 & 0,943 & 0,862 & 1,041 & 1,209 \\
\hline RSK $1 / 2 / 3$ & 1,096 & 0,961 & 0,927 & 1,058 & 1,141 \\
\hline eNOS & 1,092 & 0,987 & 0,932 & 1,030 & 1,106 \\
\hline Fyn & 1,116 & 0,977 & 0,912 & 1,041 & 1,142 \\
\hline Yes & 1,103 & 0,993 & 0,911 & 1,012 & 1,111 \\
\hline Fgr & 1,112 & 0,976 & 0,896 & 1,021 & 1,139 \\
\hline STAT6 & 1,091 & 0,992 & 0,915 & 1,006 & 1,100 \\
\hline STAT5b & 1,099 & 0,975 & 0,907 & 1,022 & 1,127 \\
\hline STAT3 (Y705) & 1,107 & 0,961 & 0,915 & 1,054 & 1,152 \\
\hline p27 & 1,105 & 0,971 & 0,913 & 1,038 & 1,138 \\
\hline PLC-ү1 & 1,088 & 0,970 & 0,925 & 1,038 & 1,122 \\
\hline Hck & 1,166 & 0,910 & 0,844 & 1,081 & 1,281 \\
\hline Chk-2 & 1,137 & 1,067 & 0,993 & 1,059 & 1,066 \\
\hline FAK & 1,185 & 0,928 & 0,859 & 1,097 & 1,278 \\
\hline PDGF R $\beta$ & 1,095 & 0,993 & 0,934 & 1,029 & 1,103 \\
\hline STAT5a/b & 1,111 & 0,983 & 0,913 & 1,031 & 1,130 \\
\hline STAT3 (S727) & 1,091 & 0,974 & 0,932 & 1,045 & 1,120 \\
\hline WNK1 & 1,213 & 0,835 & 0,845 & 1,227 & 1,452 \\
\hline PYK2 & 1,075 & 0,960 & 0,930 & 1,042 & 1,120 \\
\hline REF & 1,163 & 1,041 & 0,914 & 1,021 & 1,118 \\
\hline PRAS40 & 1,103 & 0,952 & 0,899 & 1,041 & 1,159 \\
\hline REF & 1,109 & 0,992 & 0,929 & 1,039 & 1,118 \\
\hline HSP60 & 1,324 & 0,919 & 0,675 & 0,972 & 1,441 \\
\hline REF & 1,110 & 1,000 & 0,936 & 1,039 & 1,110 \\
\hline
\end{tabular}

$<0,7 \quad 0,8 \leq x \leq 1,2$ 